\title{
Review Paper: \\ The Effect of Foot Orthosis on the Vertical Component of the Ground Reac- tion Force While Walking: A Review Study
}

\author{
Seyed Mohammad Mousavi Nodoshan ${ }^{1}$, *Ali Reza Taheri ${ }^{1}$ (i)
}

1. Department of Orthotics and Prosthetics, School of Rehabilitation Sciences, Isfahan University of Medical Sciences, Isfahan, Iran.

\begin{tabular}{|c|c|}
\hline $\begin{array}{l}\text { Use your device to scan } \\
\text { and read the article online }\end{array}$ & \\
\hline ariping & $\begin{array}{l}\text { Ctat on Mousavi Nodoshan SM, Taheri AR. [The Effect of Foot Orthosis on the Vertical Component of the Ground Reac- } \\
\text { tion Force While Walking: A Review Study (Persian)]. Archives of Rehabilitation. 2021; 22(1):10-27. https://doi.org/10.32598/ } \\
\text { RJ.22.1.3212.1 }\end{array}$ \\
\hline artag & dol'https://doi.org/10.32598/RJ.22.1.3212.1 \\
\hline
\end{tabular}

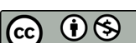

Received: 10 Jun 2020

Accepted: 29 Oct 2020

Available Online: 01 Apr 2021
Keywords:

Ground Reaction

Force, Orthotics,

Walking

\begin{abstract}
Objective Everyone is required to walk and stand for long time in daily activities, especially in the workplace. As a result, the Ground Reaction Force (GRF) on the components and joints is so great that they sometimes reach several times the weight of the body. These forces can have devastating effects on the feet and ankles. Various studies have suggested different methods and factors for reducing the GRF while walking. The purpose of this study was to identify the different causes of factors and methods affecting the GRF according to the current study.

Materials \& Methods This review study was conducted by searching the databases of ProQuest, PabMed, Web of Science, and MBS from 1995 to 2019. The keywords included 'Ground Reaction Force', foot orthosis', 'walking', 'long term standing impact force'. After reviewing the abstract and title of each study by the authors, the criteria for selecting the article were considered. At the control level of each article, based on the main design of the search, from 1 to 5 were scored as randomized control trial, prospective controlled trial (Cohort), case-control, pre/post, observational clinical consensus, respectively. The quality of the articles was evaluated and scored using the Down and Black tool.

Results Out of 82 articles, 21 articles were selected for analysis based on the criteria. Reporting scores, external validity, and internal validity varied between $4-8,3-1,5-2,6-3,7-4$, respectively. From the studies performed on the molding insoles and the change in the loading rate of the maximum vertical GRF, there is a contradiction in proving it. Investigating the effect of foot orthoses in terms of material may lead to a further reduction in plantar.

Conclusion Determining the effect of different components on GRF in the sole of the foot will help us better understand the factors involved in quality of life. The four domains of gender, post, material, and molding had different effects. Due to the lack of articles related to the study of the effect of gender and the creation of posts in the insole on the GRF can not be decisive.
\end{abstract}

\section{Extended Abstract}

\section{Introduction}

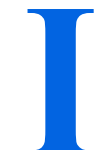

$\mathrm{n}$ a natural gait, the static phase plays an important role due to the foot contact with the ground [1] and the application of the Ground Reaction
Force (GRF) [2]. Because according to Newton's third law, the amount of this reaction force is equal to the body weight and in the opposite direction to the force that the body exerts on the ground as a result, the articulated moments created are directly related to the amount of GRF and the vertical distance from the center of force to the joint [3]. The vertical component of ground reaction force to the joint force is of greater

\section{* Corresponding Author:}

Ali Reza Taheri, PhD.

Address: Department of Orthotics and Prosthetics, School of Rehabilitation Sciences, Isfahan University of Medical Sciences, Isfahan, Iran. Tel: +98 (913) 3189373

E-Mail: taheri@rehab.mui.ac.ir 
biomechanical importance due to its greater value than the anterior-posterior and internal-external components, as well as its shape of stability in individuals (ground reaction force increases by up to $120 \%$ of body weight from the static phase $[4,5]$. Improper absorption of the impact forces of the foot on the ground is one of the biomechanical parameters that can cause problems for the foot due to the misalignment of different parts in the lower limb. According to the graph ofthe graph ground reaction force (Figure 1) there are to pwo peak points, there are two peak points $[6,7]$; the first peak force is produced by the foot hitting the ground, and the second peak force is produced by the pressure of the toe to the ground [2, $8]$. The ground reaction is a good criterion for identifying and classifying people based on the pattern of forces when walking, which can be different in the peak of forces.

In previous studies, the relationship between increasing the vertical component of the GRF and increasing the risk of injury to the body [4-6] has been investigated. If these forces are repeated, there is a possibility of joint damage, especially in continuous weighting [9-14].

Changes in the insoles can also change the lever arm of the GRF [15]. Although based on biomechanical principles and several studies, the use of insoles reduces the first peak of reaction force and loading rate, other studies have reported that insoles increase the first peak of reaction force in healthy people. Even in a study, it has been shown that different insoles have little effect on the kinetic variables of people while walking [16-18]. However, part of these differences is due to the kinetic and kinematic effects of displacement of the pressure center [19].

Effect of factors, such as insole material on the vertical reaction force of the earth has been considered by researchers [15] in some studies. With decreasing insole stiffness, the vertical reaction force of the earth increased [20]; however, in some studies, no significant difference was observed in the effect of insole stiffness [14]. It is unavoidable that vertical forces can be reduced by making interventions in shoes and insoles for easier walking, but it is worth considering that in various studies, there is insufficient instability in different methods and factors on the reaction forces. This study aimed was done to determine the various factors and methods affecting the vertical components of reaction force and loading rates when walking in healthy individuals.

\section{Materials and Methods}

The present review study was conducted with extensive search in databases, such as ISI web of knowledge, Embase, PubMed, and Proquest from 1995 to 2019 using the keywords of ground reaction force, foot orthosis, walking, and impact force of long-term standing. To find more relevant articles, the sources of the selected articles were also examined. After reviewing the titles and abstracts of articles focusing on orthoses and GRF by the authors, in addition to answering the characteristics and questions of researchers in the next phase, the following inclusion criteria were considered for selecting and reviewing articles:

\section{The article must be in English.}

\section{The article must be published from 1995 onwards.}

3. The parameter measured in each article should be at least one of the parameters considered in the study (walking, foot orthoses, GRF, and vertical components).

The control level of each article was rated 5 to 1 for randomized control trials, prospective controlled trials (cohort), case-control studies, studies with pre/post-test design, and observational consensus-based clinical studies, respectively. Then, the quality of the articles was examined by the Down and Black tool and Pedro index [21, 22]. It should be noted that the accuracy and validity of this test have been proven as a criterion for reviewing and evaluating the quality of research studies. The quality of each article was evaluated separately by two experts, and then, using the Pearson correlation test, the relationship between the results was checked through SPSS V 16 software.

\section{Results}

Of the 82 articles obtained, 21 articles met the inclusion criteria (Figure 1). According to Table 1, four domains, including the effect of molding, post, material type, and gender were obtained. Seven articles examined the effect of molding on the GRF, and five articles, directly and indirectly, had examined the effect of the insole with a post on the GRF. The effect of material type on the GRF had been investigated in seven studies. Regarding gender, only two articles had focused on GRF without orthoses.

Some studies on the GRF had focused on unhealthy humans. There was an obvious discrepancy between the molding insoles and the change in the maximum vertical force of the ground reaction loading rate. Regarding the effect of the material, it can also be said that it may lead to a further reduction in the pressure of the sole of the foot. Regarding gender, although women have a higher loading rate in the GRF than men without the use of a device, no study was found on the use of insoles and the effect of gender.

According to the results of the quality evaluation of articles, about half of the studies were randomized clinical 


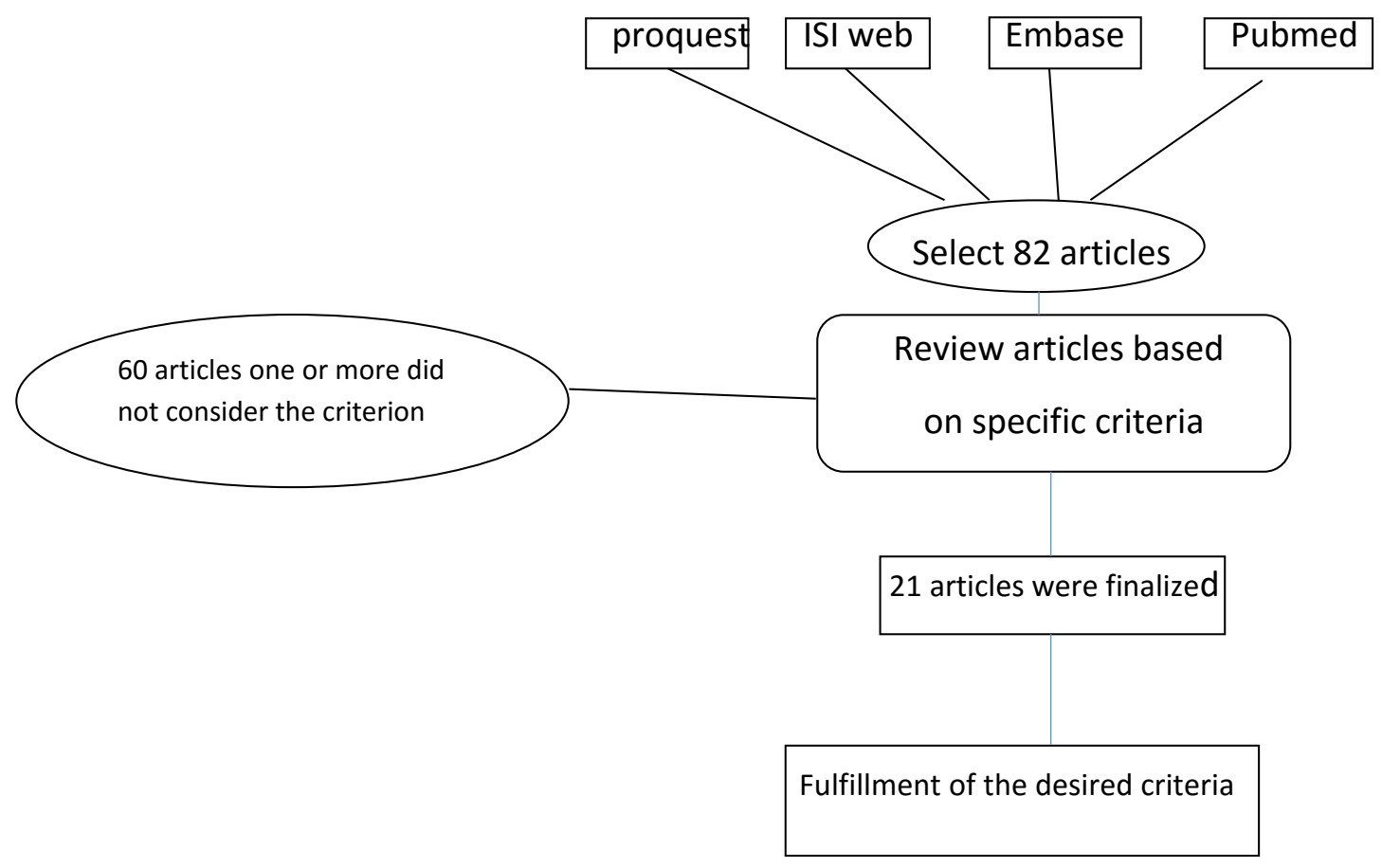

Figure 1. Diagram of how to select the appropriate articles for this research

Rehabilitation

trials (rate of 5). The high scoring, external validity, internal validity, and Pedro index all indicated the relatively good level of trust and credibility of the articles.

\section{Discussion and Conclusion}

This study was done to investigate the changes in GRF produced by foot orthoses due to different effective factors and methods. Determining the effect of different components on GRF in the foot will help us in prescribing orthoses to increase the quality of life of people. The results showed that the four domains have different effects on the vertical component of ground reaction force.

\section{Impact of molding}

One of the features of foot molding for making insoles and shoes is that it creates a relatively complete contact with the sole of the foot, which is effective in reducing the forces and its proper distribution [6]. Molded orthoses with more contact between the foot and orthoses increase shock absorption and decrease GRF. A reduction in the first peak of the vertical ground reaction force by the semi-rigid insole has been able to prevent joint destruction damage to some extent [23] and on the other hand, reduce the pressure of the heel and the inner area of the front of the foot and the impact of forces to a quarter percent compared with without insole [6]. Significant reduction in leg fatigue after wearing these insoles [24] and a reduction in loading rate are evident $[23,25]$. In contrast to these studies, Sloss reported a 3\% $-5 \%$ increase in the vertical force component in the first peak by carbon-molded orthoses [26] or MacLean did not observe significant changes in maximum impact force and loading rate with this type of orthosis [27]. The reason why these two studies are different despite proving the biomechanical alteration of the foot in the correct position with the insole may be related to other factors, such as the material and shape of the orthosis.

\section{Post effect}

One of the purposes of using foot orthoses is to place the foot and lower limb in a better functional position while maintaining a proper alignment and to improve the absorption impact $[16,28]$. In a study combining the two methods of molding and post, a decrease in vertical loading rate was observed. Also, a significant difference in the reduction of the maximum vertical reaction force of the earth and the loading rate of the vertical reaction force of the earth during the static phase was proved by post [29-31]. Rome et al., along with the post, changed the insole material and density and the shape of the insole, and subsequently observed a decrease in loading change [32].

\section{Impact of soft materials}

Insole material is one of the factors with a great impact on changing reaction forces. Visco-elastic insoles, by following the physiological structure of the body in the face of exces- 
Table 1. Analysis of articles related to orthotics and its effect on ground reaction force

\begin{tabular}{cccc}
\hline Area & Source & $\begin{array}{c}\text { Author and Year } \\
\text { of Publication }\end{array}$ & Subjects and Methodology \\
\hline
\end{tabular}

\section{Alirezaie 2017}

The soft-

ness of

the floor

[39] Razeghi 2003

Creaby 2011

Miller 1996

[34]

OLeary 2008

Assessing 16 people with custom mold polyurethane insoles and without insoles to indicate how to affect impact absorption

[25]

Maundermann 2003

in 21 people

Comparison of ground reaction force variables with the insole and cushioned hee and insole on 45 people

Investigation of the effect of Hill Cup insole and insole on the force loading rate in 14 people

Investigation of ground reaction force on 25 people with semi-rigid orthoses without orthoses and standard shoes

nvestigating the effect of post and molding
in 21 people

Survey of 11 healthy men with shoes and

[23] Eslami M, 2009 boots with orthoses on the ground reaction force

Evaluation of comfort of 3 types of heel height and 4 types of insoles inside the shoe on the distribution of sole pressure and impact force in 10 women

The effect of molded orthoses on the kine-

[28] MacLean 2006 matics of 15 healthy individuals with shoes, and shoes and insoles with posts

Investigation of 10 insoles and three foot orthoses made of polypropylene, carbon on the vertical force of ground reaction

Investigating the effect of molded orthoses
MacLean 2006 on 15 healthy people with shoes, and shoes and insoles with posts
There was a significant difference between the three types of insoles in the first peak of the vertical force of the earth, and in the subsequent peaks of this significant difference, between the viscoelastic insole and other insoles.

The effect of shoes with soft foam insoles on foot discomfort and fatigue

- Ground and shoe conditions and prolonged standing affect a person's lower limbs.

- Increasing the vertical load of GRF insole with soft shoes

- Reducing vertical loading with hard cushion insole

The vertical force on the silicone hill pad does not reduce the expected impact force, and the hypothesis that the shock absorption is better by the Hill Cushion is not easily proven.

Increased loads and forces on the knee are involved in the spread and progression of knee injuries.

The maximum impact force is enough to reduce the vertical component of ground reaction force in the first $20 \%$ of the static phase

- The shock absorber insole significantly reduces the maximum impact force of the ground on the shoe.

- Reducing the reaction force loading rate compared with shoes

- Increasing the vertical loading rate with the post and increasing the shock absorption with the molded orthosis

- Reducing the vertical loading rate by molding and combining molding and post

$6 \%$ reduction of the semi-rigid insole in the first peak of the vertical reaction force of the ground - Limiting the back foot movements and rotation of the tibia and correcting the torque arm with the orthosis

Reducing heel pressure and impact force with heel pads in high heels

No significant change in maximum impact force and loading rate with the orthosis

Increasing the vertical component of the force in the first peak with the orthosis and little changes in the second peak

There are no significant changes in maximum impact force and loading rate with orthoses 


\begin{tabular}{|c|c|c|c|c|}
\hline Area & Source & $\begin{array}{l}\text { Author and Year } \\
\text { of Publication }\end{array}$ & Subjects and Methodology & Conclusion \\
\hline \multirow{5}{*}{ Post } & [32] & Rome 2016 & $\begin{array}{l}\text { Evaluation of the effects of high-density } \\
\text { polyethylene ortho ethylene-vinyl acetate } \\
\text { with internal longitudinal arch, long hill cap, } \\
\text { and post in } 41 \text { patients }\end{array}$ & $\begin{array}{l}\text { This combination of orthosis material was very } \\
\text { effective in changing the load and reducing pain }\end{array}$ \\
\hline & [29] & Laughton 2003 & $\begin{array}{l}\text { Investigation of } 15 \text { healthy individuals with } \\
\text { insoles and orthoses with post and without } \\
\text { orthoses on the ground reaction force }\end{array}$ & $\begin{array}{l}\text { Significant reduction in maximum vertical force } \\
\text { and loading rate with post orthoses }\end{array}$ \\
\hline & [30] & MacLean 2008 & $\begin{array}{l}\text { Examination of } 15 \text { women with insoles in } \\
\text { two positions with semi-rigid and low or- } \\
\text { thosis and without orthosis }\end{array}$ & Reducing loading rates during insole walking \\
\hline & [43] & Park 2018 & $\begin{array}{l}\text { Evaluation of vertical forces in } 40 \text { people } \\
\text { with orthoses and insoles based on gender }\end{array}$ & $\begin{array}{l}\text { Ground reaction force loading component is } \\
\text { higher in women than men at different speeds }\end{array}$ \\
\hline & [42] & Chiu 2006 & $\begin{array}{l}\text { Investigating the effect of walking speed } \\
\text { and gender of } 30 \text { people with bare feet on } \\
\text { the ground reaction force }\end{array}$ & $\begin{array}{l}\text { The short stature of women compared with men } \\
\text { to achieve a constant speed increases the maxi- } \\
\text { mum loading rate }\end{array}$ \\
\hline
\end{tabular}

sive forces, have the best efficiency in absorbing impact and reducing the reaction forces of the ground [9]. Supporting softness next to a high heel shoe is recommended to control lateral movements and prevent foot injury $[33,34]$. In some studies, a significant difference in fatigue and discomfort of people wearing visco-elastic insoles was observed [14, $21,29]$. The reduction of the maximum impact force of the ground and the loading rate has been more than the use of shoes [35], especially at the beginning of the static phase [36]. Alirezaie announced a reduction in the vertical load of GRF with a hard cushion insole and without soft shoes [37].

Contrary to these reports, some studies did not change the characteristics of the GRF in the use of viscoelastic insoles [38] and even soft orthoses, such as silicone pads did not reduce the impact force as expected, and we cannot support the hypothesis that heel cushions provide better shock absorption [39] or orthoses with softer materials than semi-rigid ones do not necessarily lead to a further reduction in sole pressure [40].

This difference in studies suggests that changes in gait parameters, including the GRF, depend on various factors, such as age, type of insole, the material of the shoe, and even the longevity of the soft insole.

\section{The effect of gender}

Although the gait parameters in men and women are somewhat different, the gait symmetry, which indicates the level of GRF in each limb, is slightly different between both genders [41]. Min-Chi Chiu stated that because women are shorter than men, they need more muscle activity to achieve a predetermined constant speed, which increases the maxi- mum loading rate compared with men [42]. In one study, the loading component of GRF was higher in women than men despite different speeds [43].

Finally, to achieve more accurate results and resolve the contradiction between studies on the effect of material type and orthosis type mechanism on the vertical components of the reaction force, more research should be done with modern motion analysis systems [44]. One of the reasons for comfort and improvement of life indicators is related to the effect of insole comfort in terms of gender and proper distribution of GRF in the balanced use of leg muscles and absorption of blows caused by walking on feet and ankles. GRF may be affected by foot orthosis, indicating the existence of various and influential factors, such as age, gender, and orthosis preparation method, that should be further studied.

\section{Conclusion}

Determining the effect of different components on GRF in the sole of the foot can help us to better prescribe and understand the factors affecting the quality of life. Our results indicated that factors, such as gender, post, molding, and type of material, despite the effect on the GRF, provide different sign. Different variables should be considered in designing and manufacturing foot orthoses using different methods, and also changes in shoes and orthoses are needed o be considered to change the GRF. 
Ethical Considerations

Compliance with ethical guidelines

This article is a meta-analysis with no human or animal sample. There were no ethical considerations to be considered in this research.

\section{Funding}

This research did not receive any grant from funding agencies in the public, commercial, or non-profit sectors.

Authors' contributions

Conceptualization and supervision: Ali Reza Taheri; Methodology: Mohammad Mousavi; Investigation, writing - original draft, and writing - review \& editing, data collection, data analysis: Both author.

\section{Conflict of interest}

The authors declared no conflict of interest.

Acknowledgments

We would like to thank MS Rahmani, the head of the library of the Faculty of Rehabilitation Sciences, for providing us with resources. 
This Page Intentionally Left Blank 


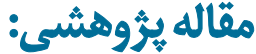

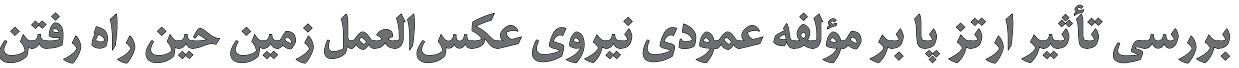 \\ سيد محمد موسوى ندوشن'، "عليرضا طاهرى'
}

ا. كروه ارتوز و يروتز، دانشكده علوم توانبخشى، دانشكاه علوم يزشكى اصفهان، اصفهان، ايران.

\begin{abstract}
حكSי

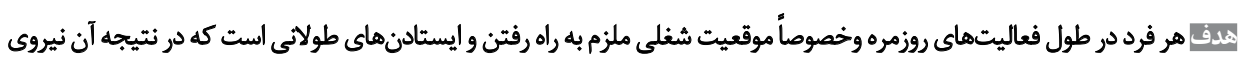

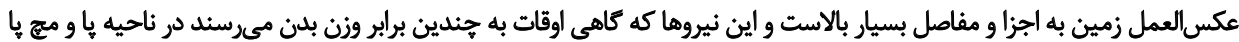

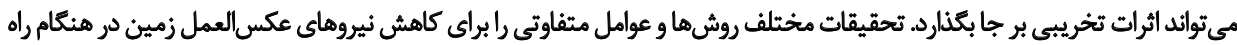

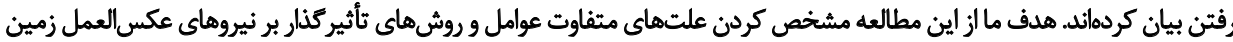

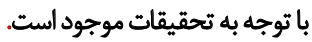

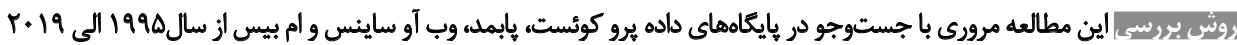

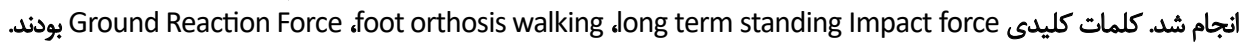

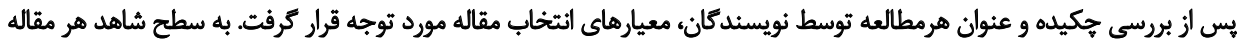

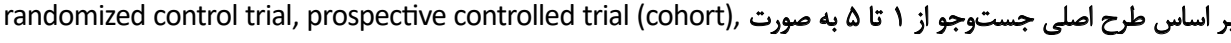
case-control, pre/post, observational consensus clinical and

$$
\text { Down and Black }
$$

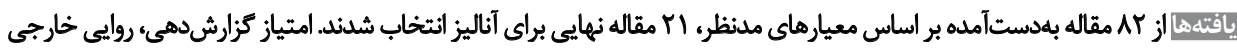

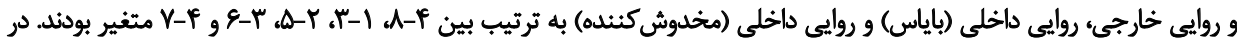

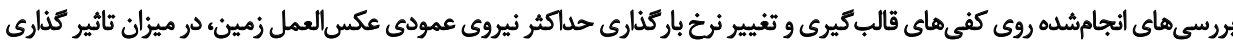

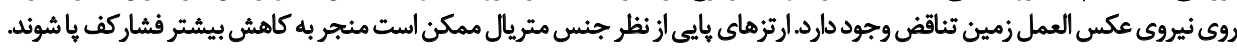

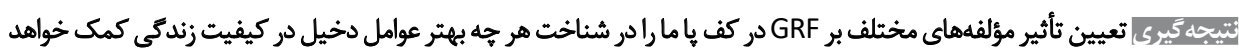

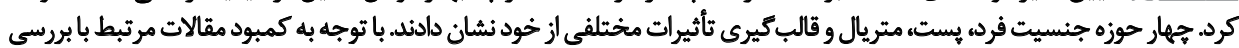

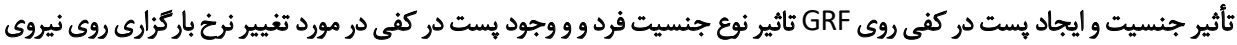

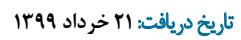

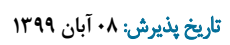

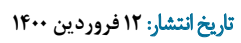

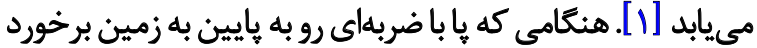

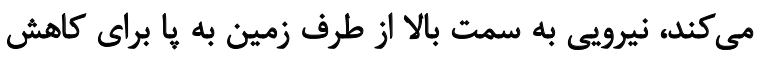

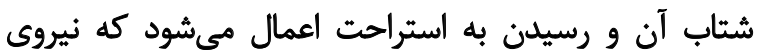

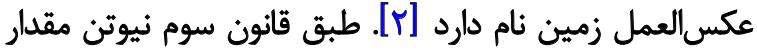

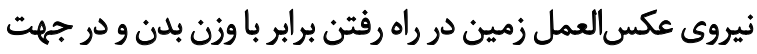

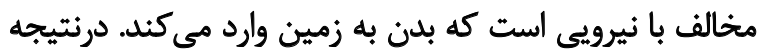

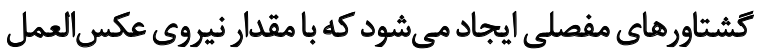

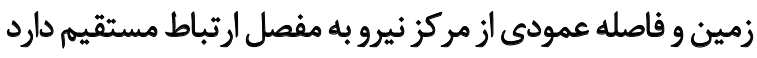

\section{Stance \\ 3. Swing}

1. Gait

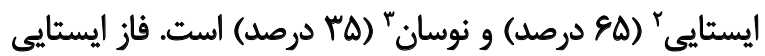

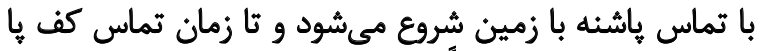

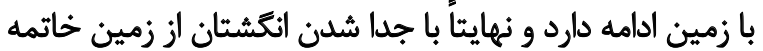

: زويسينده مسيئول:

دكتر عليرضا طاهرى نشاني: اصفهان، دانشكاه علوم بزئشكي اصفهان، دائشكده علوم توائبخشي، كروه ارتوز و بروتزو. تلفن: taheri@rehab.mui.ac.ir رايانامهن 


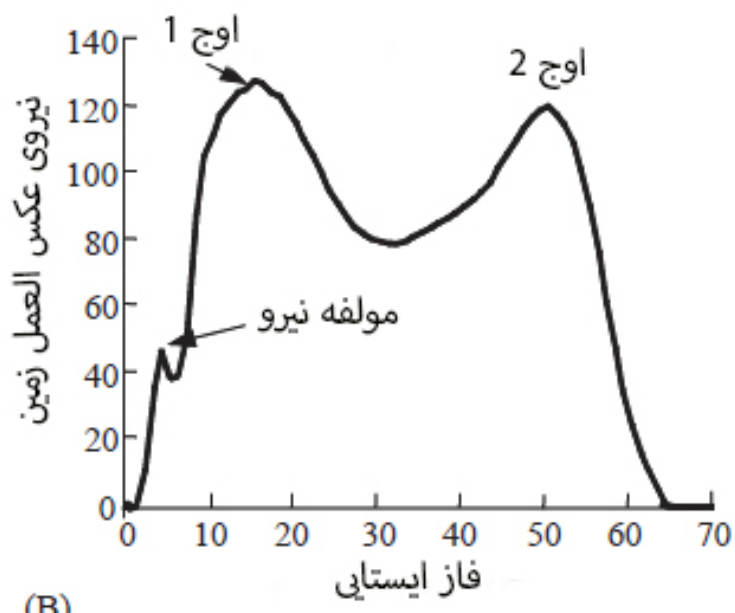

(B)

تصوير ا. نمودار توليد نيروى عكس العمل زمين در حركات ارادى توانبننتى

مطالعات ديكرى هم كزارش كردند كه كفى موجب افزايش

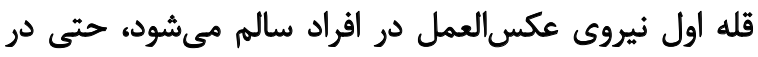

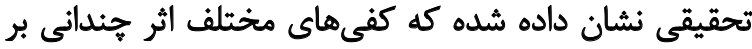

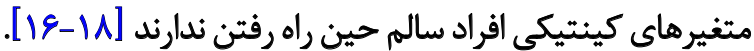

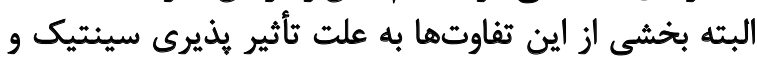

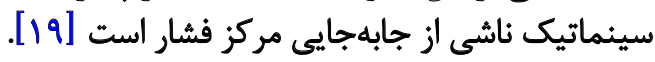

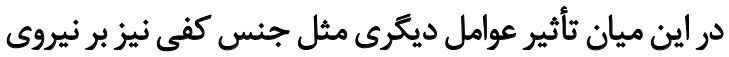

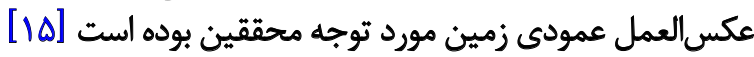

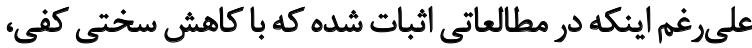

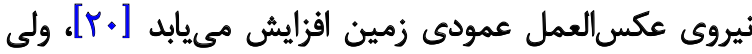

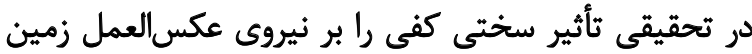

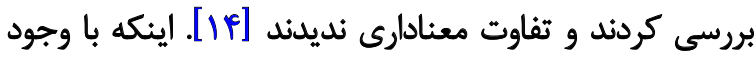

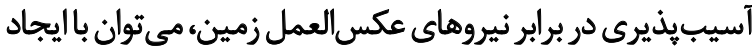

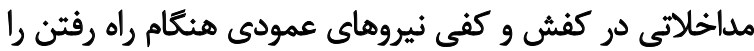

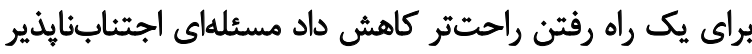

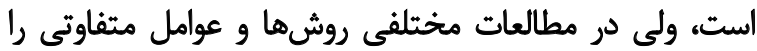

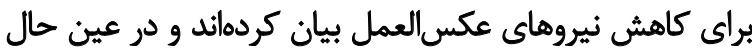

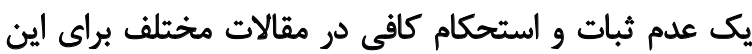

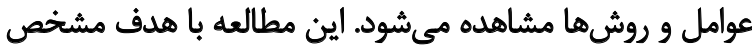

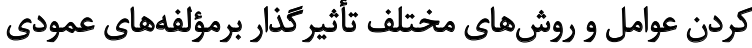

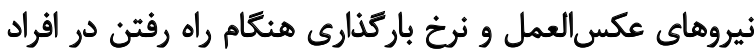

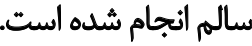

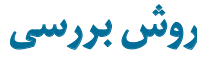

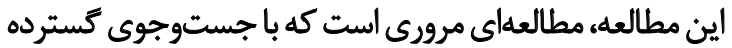

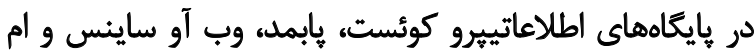

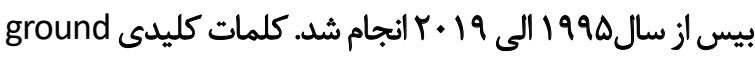
reaction force foot orthosis ،walking dong term
[ץ]. مؤلفه عمودى نيروى عكس العمل زمين به دليل مقدار بيشتر

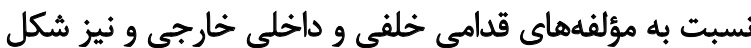

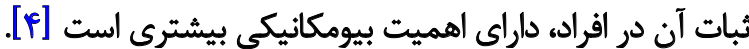

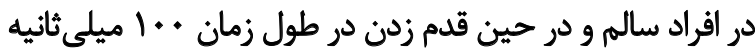

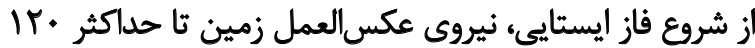

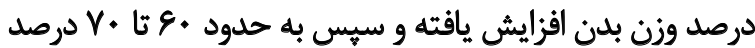

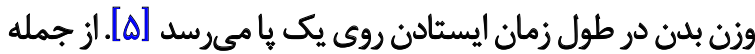

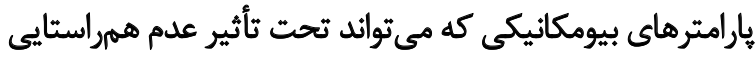

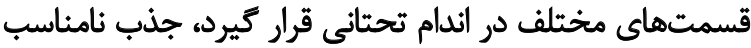

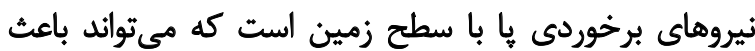

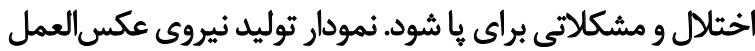

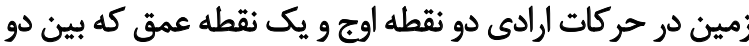

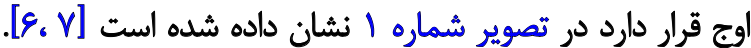

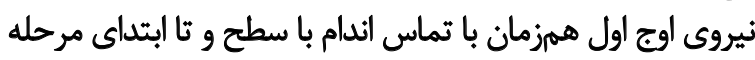

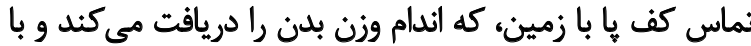

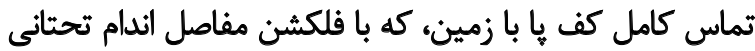

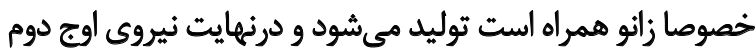

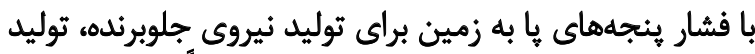

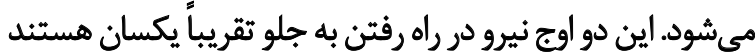

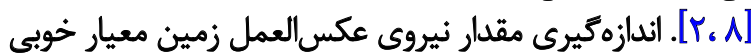

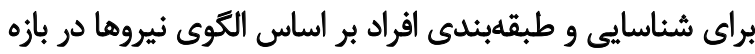

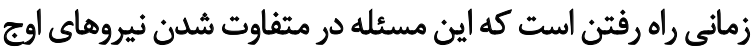

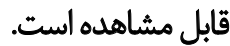

در بخشى از مطالعات بيشين ارتباط بين افزايش مؤلفه عمودى

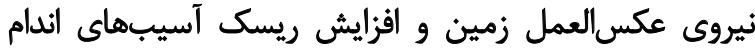

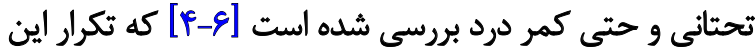

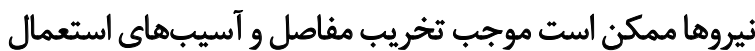

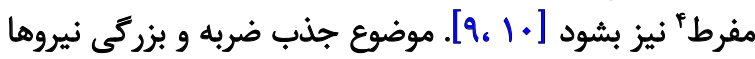

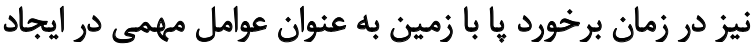

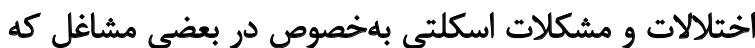

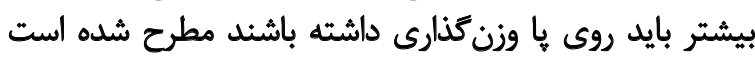

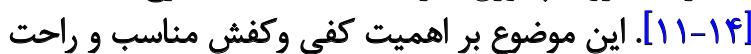

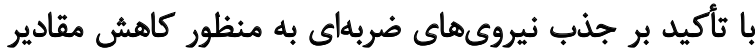

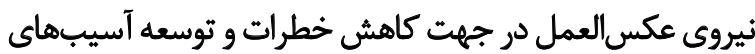

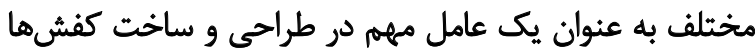
مورد توجه طراحان و سازندكان كفش قرار كرفته است.

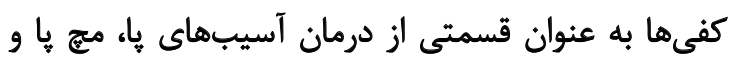

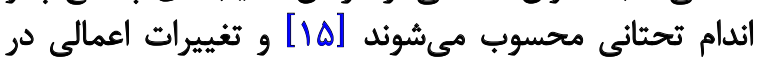

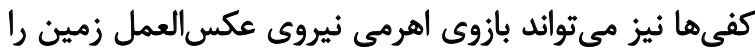

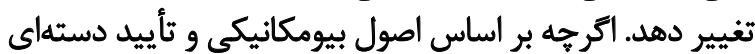

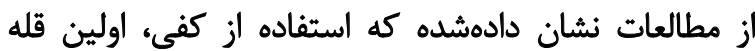

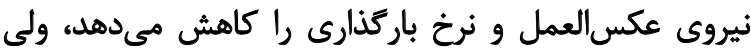

\section{Overuse injuries}




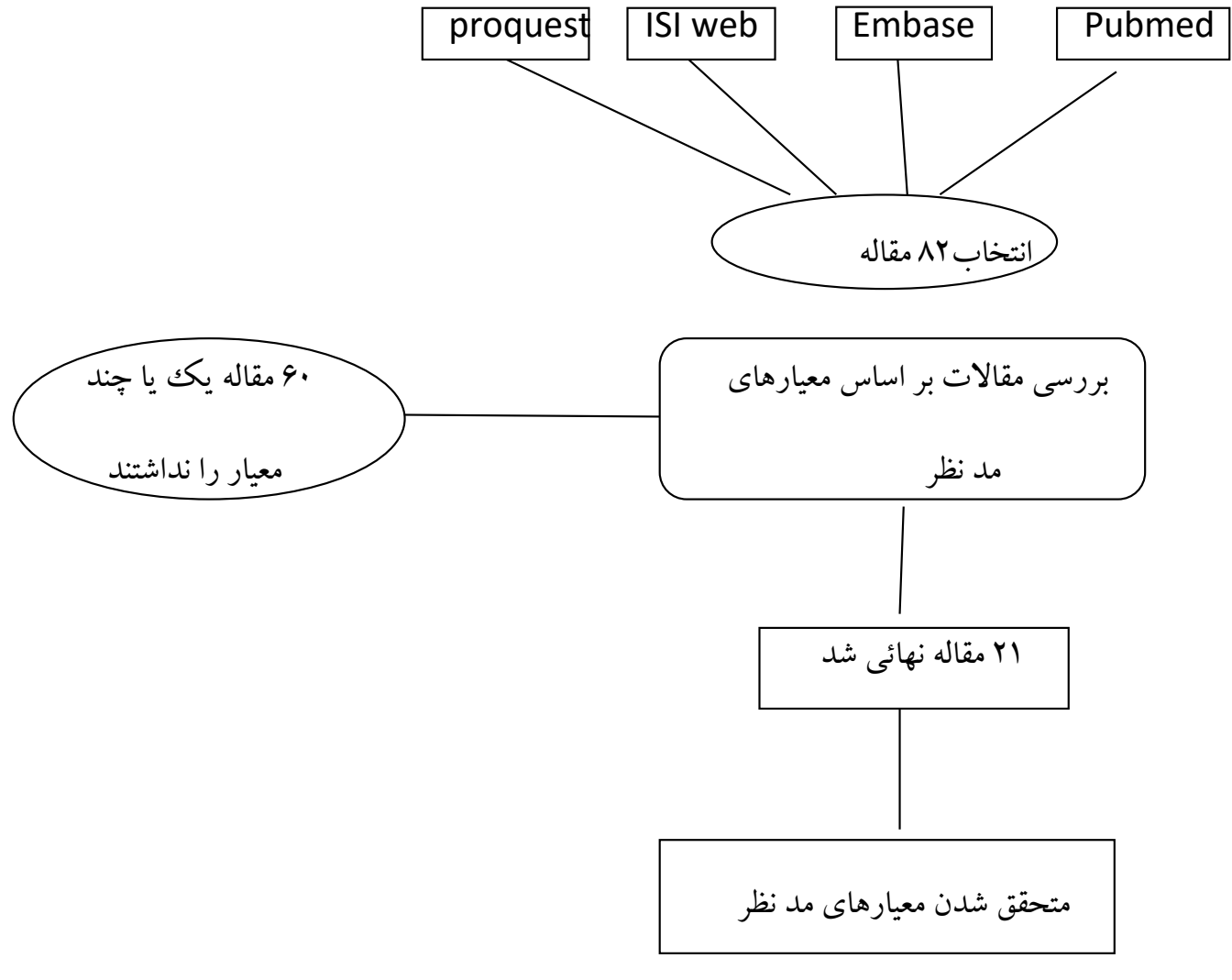

توانبخنتى

تصوير r. نمودار نحوه انتخاب مقالات مناسب اين تحقيق

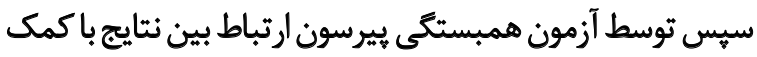

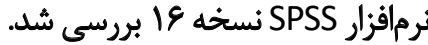

Lodị

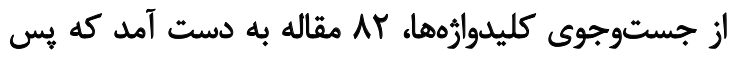

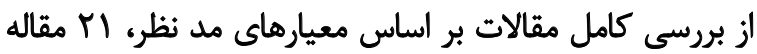

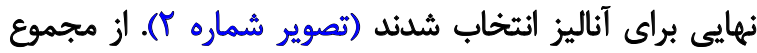

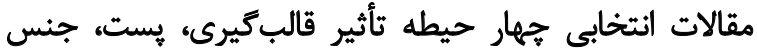

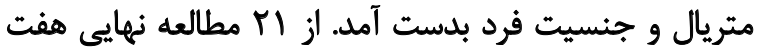

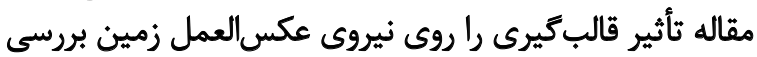
كرده بودند. از ينج مقاله كه ثأثير كفى داري داري

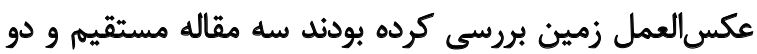

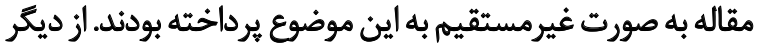

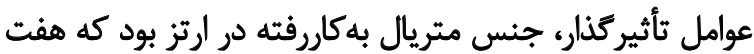

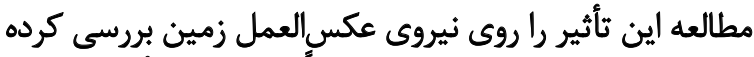

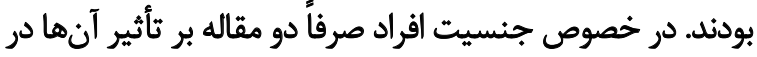

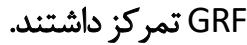

احرحه اغلب مثالات نيروى عكسالعمل زمين را روى عوامل مختلف بررسى كرده بونند، ولى بخشى از مطالعات روى انسان

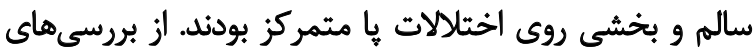

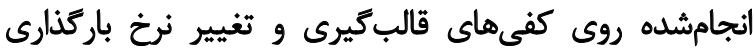

Standing Impact force كستردكى بيشتر محدوده جستوجوء از منابع مقالات كشفش جشده

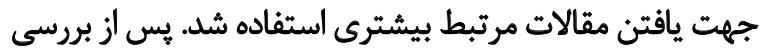

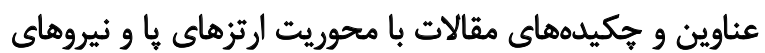

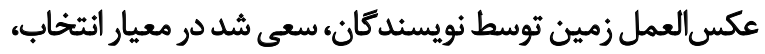

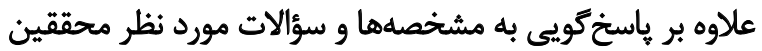

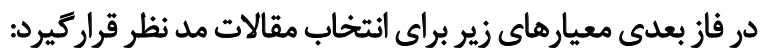

$$
\text { I. ا. مقاله حتماً به زبان انكليسى باشد. }
$$

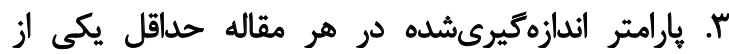

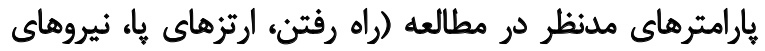
عكس العمل زمين، مؤلفههاى عمودى) باشدي.

به سطح شاهد هر مقاله بر اساس طرح اصلى جستوجو از

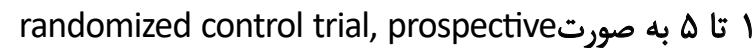
controlled trial (cohort), case-control, pre/post, obشئ به servational consensus clinical and

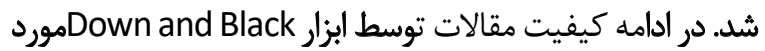

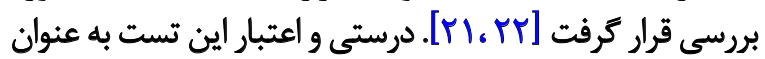

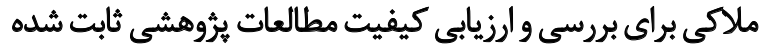

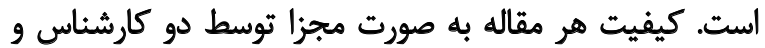


جدول (. تحليل مقالات مرتبط با ارتز و تأثير آن روى نيروى عكس العمل زمين

\begin{tabular}{|c|c|c|c|c|c|}
\hline منيع & نويسنده و سال انتشار & موضوع & مداخله & روش كار و اندازهكيرى & تثيجهكيرى \\
\hline [ma ] & Shiba, 1995 & 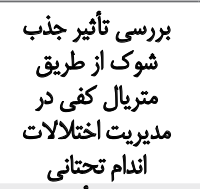 & 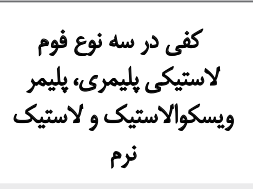 & أنجام تست روى شائزده فرد & 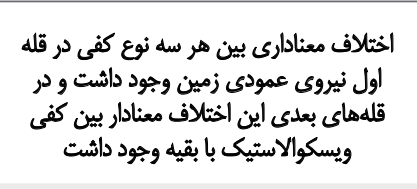 \\
\hline$[1 F]$ & Lin, 2012 & 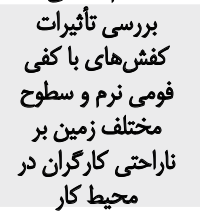 & 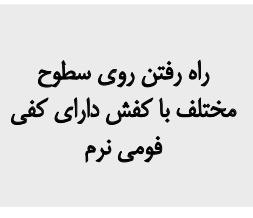 & 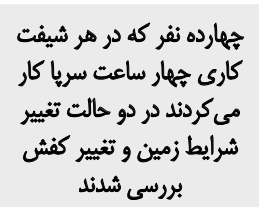 & 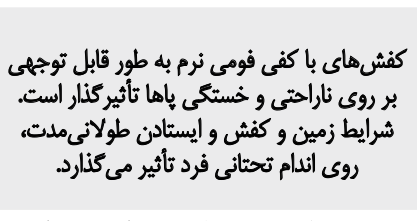 \\
\hline [ro] & Sobel, 2001 & 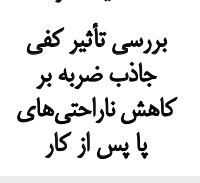 & كفى قالب كيرى شلده & 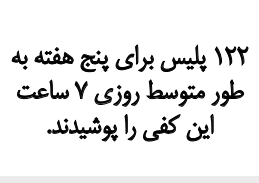 & 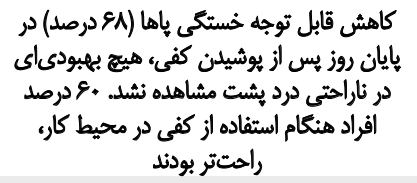 \\
\hline [世ㅅ] & Alirezaie, 2017 & 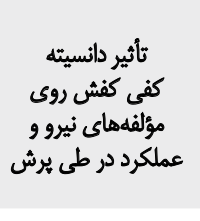 & كفى نرمو ونيمهسخت و & 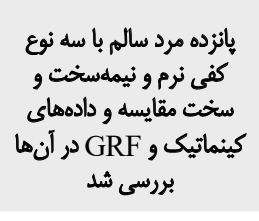 & 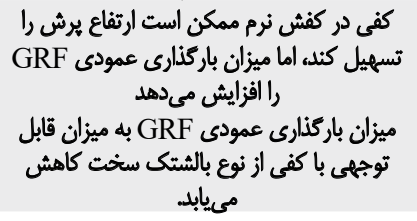 \\
\hline$\left[*_{*}\right]$ & Razeghi, 2003 & 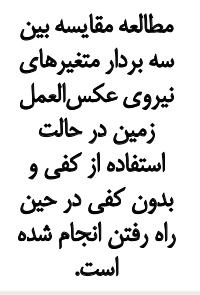 & $\begin{array}{l}\text { 1. Heel posting } \\
\text { 2. Heelcushioning } \\
\text { 3. No insole }\end{array}$ & 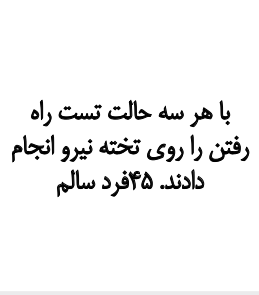 & 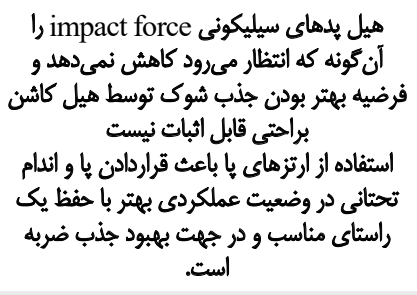 \\
\hline [re] & Creaby, 2011 & 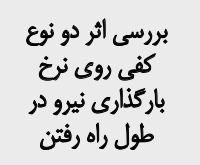 & $\begin{array}{l}\text { 1. No insole } \\
\text { 2. Flat material } \\
\text { insole } \\
\text { 3. Heel-cup insole }\end{array}$ & 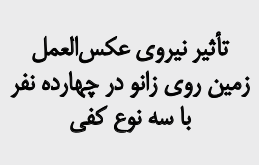 & 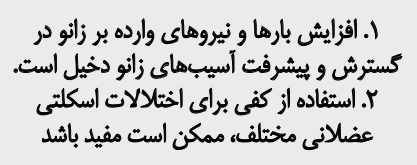 \\
\hline [re] & $\begin{array}{l}\text { Maundermann, } \\
2003\end{array}$ & 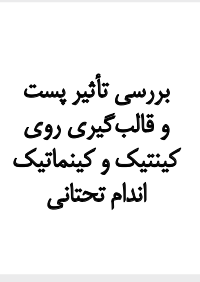 & $\begin{array}{l}\text { 1. Medial posting } \\
\text { 2. Custom-molding } \\
\text { 3. Medial posting } \\
\text { and } \\
\text { custom molding }\end{array}$ & 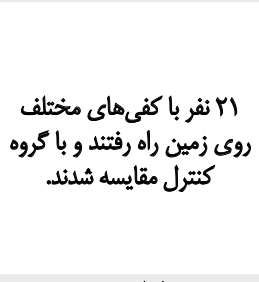 & 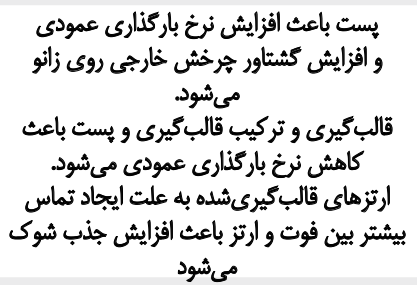 \\
\hline$[\mathrm{HT}]$ & Chiu, 2006 & 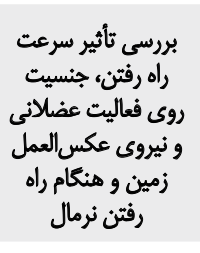 & راه رفتن با ياي برهنه & 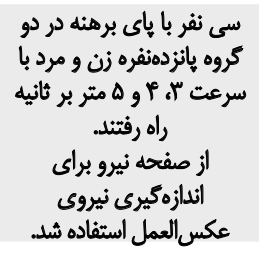 & 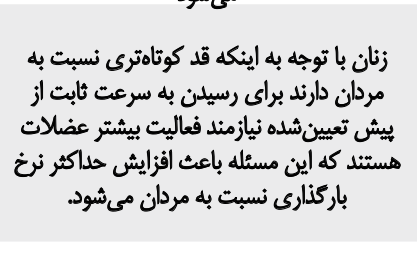 \\
\hline$[\mathrm{r}]$ & Eslami, 2009 & 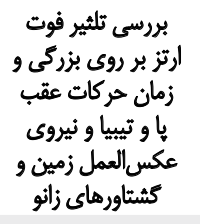 & 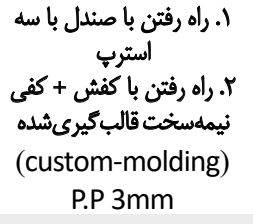 & 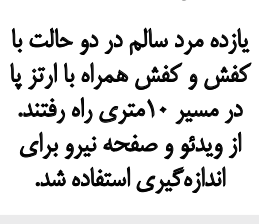 & 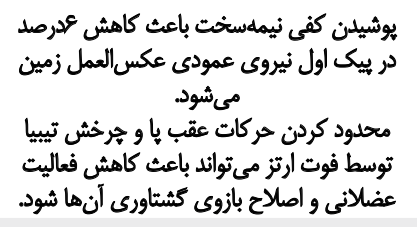 \\
\hline$[\% 1]$ & Tenten, 2019 & 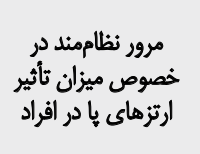 & 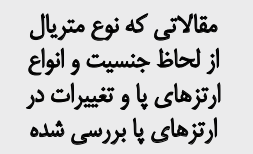 & 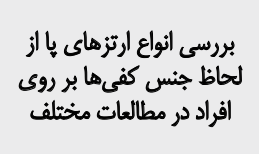 & 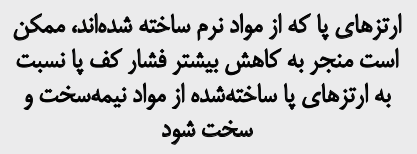 \\
\hline
\end{tabular}




\begin{tabular}{|c|c|c|c|c|c|}
\hline منيع & نويسنده و سال اتتشار & موضوع & مداخله & روش كار و أندازهيرى & نتيجهيرى \\
\hline [\&] & Yung-Hui, 2005 & 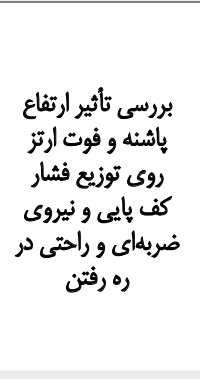 & 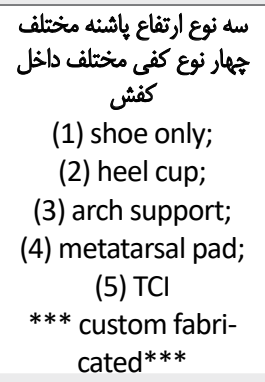 & 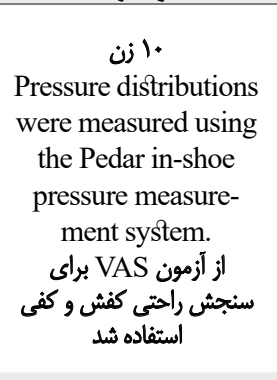 & 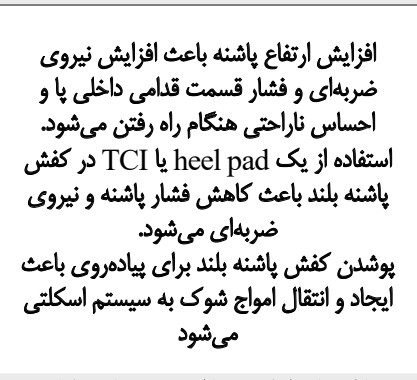 \\
\hline$\left[\mathbb{r}^{\mathrm{c}}\right]$ & OLeary, 2008 & 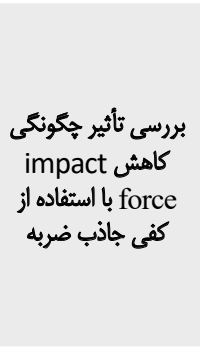 & 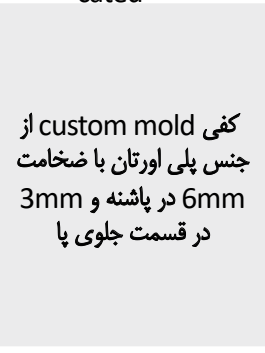 & 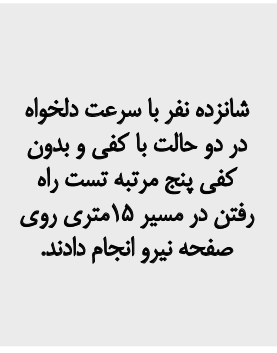 & 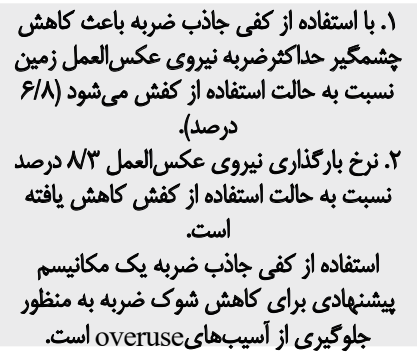 \\
\hline [थT] & Rome, 2016 & 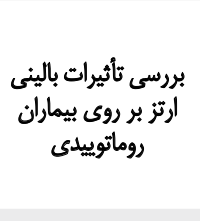 & $\begin{array}{l}\text { foot orthoses } \\
\text { (CMFOs) and simple } \\
\text { insoles (SIs }\end{array}$ & 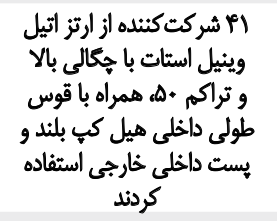 & 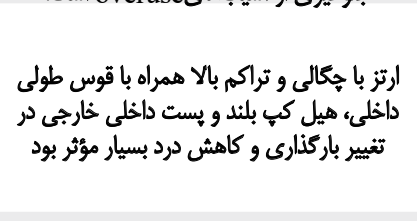 \\
\hline$[\pi]$ & King, 2002 & 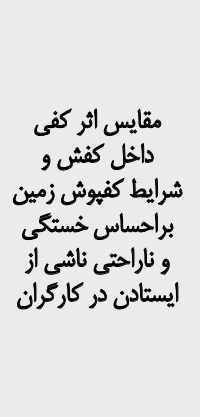 & كفى هاى ويسكوالاستيك & 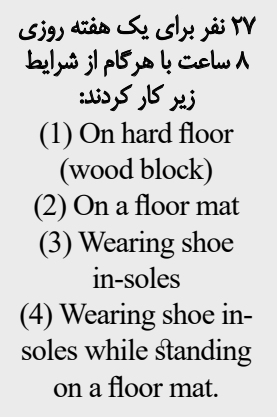 & 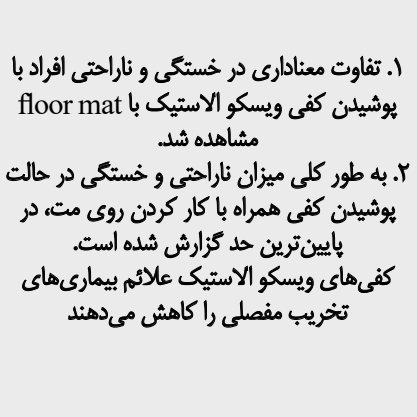 \\
\hline$[\mathrm{IV}]$ & Nester, 2003 & 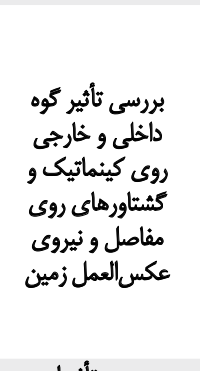 & $\begin{array}{l}3 \mathrm{~mm} \text { basemate- } \\
\text { rialmanufactured } \\
\text { fromEVA }\end{array}$ & 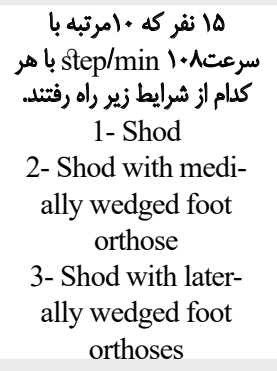 & 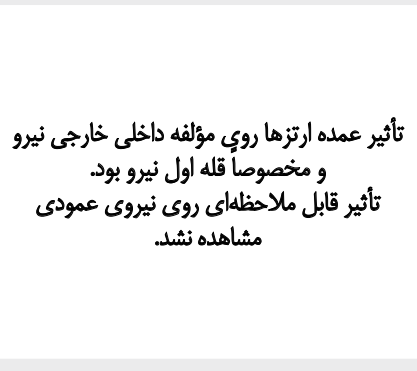 \\
\hline [ri)] & MacLean, 2008 & 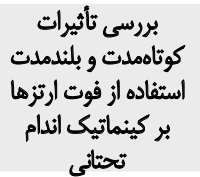 & $\begin{array}{l}\text { Semi-rigid Custom } \\
\text { Foot Orthotic (CFO) } \\
\text { post }\end{array}$ & 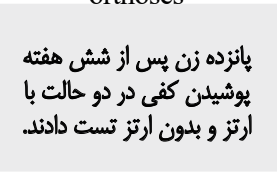 & 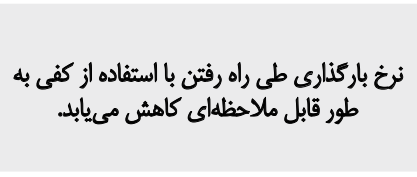 \\
\hline$[\mathrm{V}]$ & Miller, 1996 & 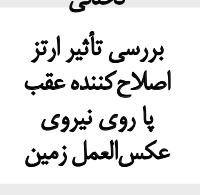 & $\begin{array}{l}\text { 1. Semirigid func- } \\
\text { tional rearfootor- } \\
\text { thoses } \\
\text { 2. Standard shoe } \\
\text { only }\end{array}$ & 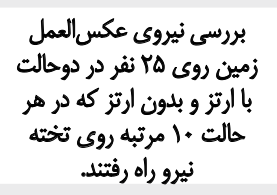 & 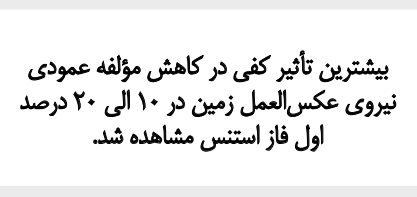 \\
\hline$[*]$ & Park, 2018 & 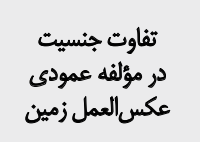 & $\begin{array}{l}\text { Foot Orthoses } \\
\text { (CMFOs) and Simple } \\
\text { Insoles (SIs) }\end{array}$ & 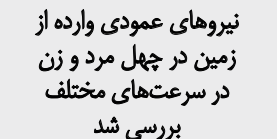 & 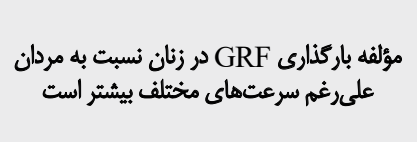 \\
\hline
\end{tabular}




\begin{tabular}{|c|c|c|c|c|c|}
\hline مثيع & نويسنده و سال انتشار & موضوع & مداخله & روش كار و اندازهكيرى & نتيجدكيرى \\
\hline$[r \cdot]$ & Laughton, 2003 & 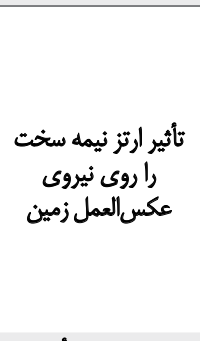 & 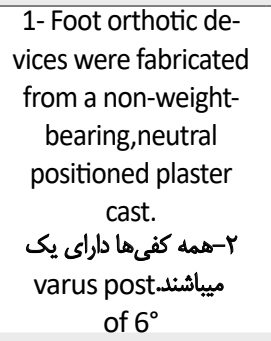 & 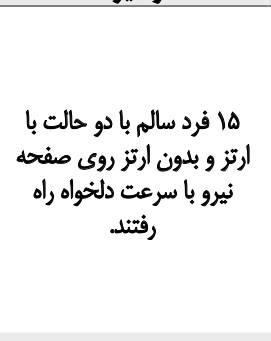 & 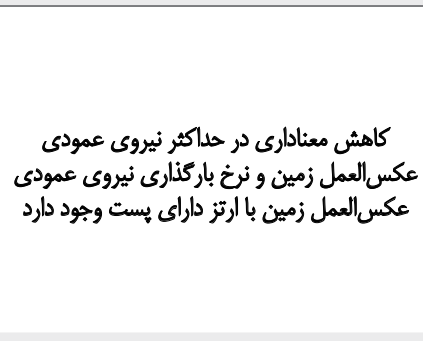 \\
\hline$[\mathrm{YA}]$ & MacLean, 2006 & 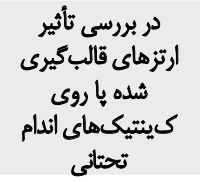 & $\begin{array}{l}\text { 1- Shoe only } \\
\text { 2- Shoe + custom } \\
\text { foot orthotic }\end{array}$ & 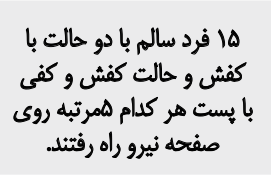 & 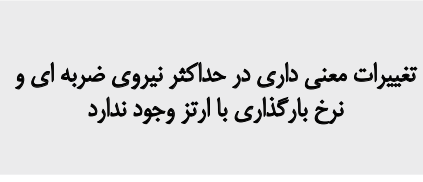 \\
\hline$[\mathrm{YV}]$ & Sloss, 2001 & 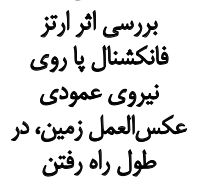 & 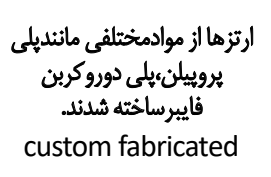 & 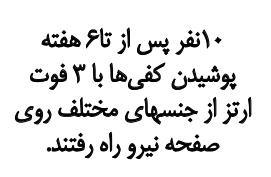 & 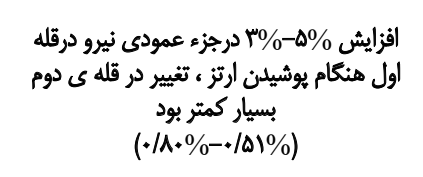 \\
\hline
\end{tabular}

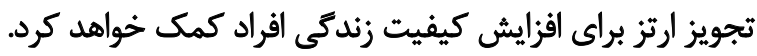

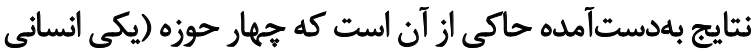

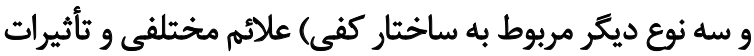

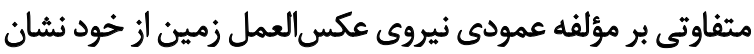
مى نهند.

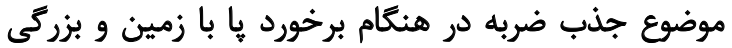

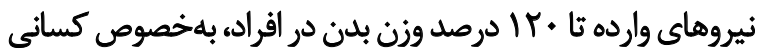

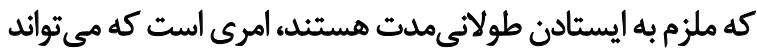

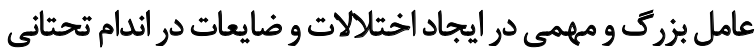

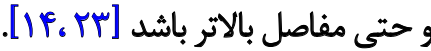

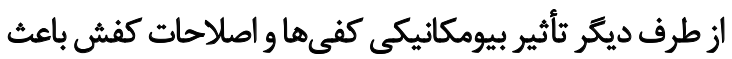

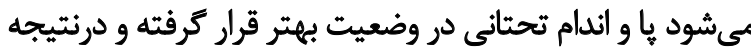

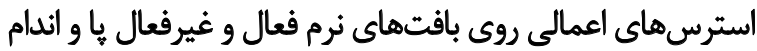

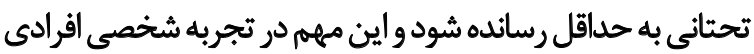

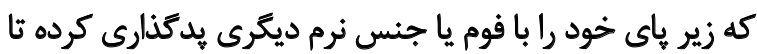

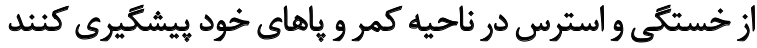

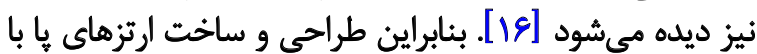

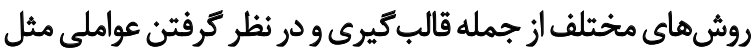

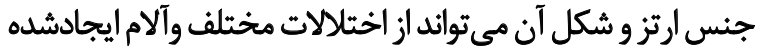

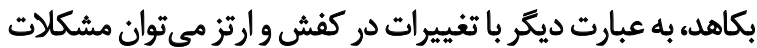

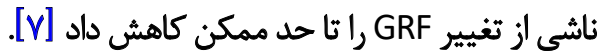

$$
\text { ثأثير قالب كيرى بر ثيروى عكس العمل زمين }
$$

از ويرُكى هاى قالب تيرى از با براى ساخت كفى و كفش اين

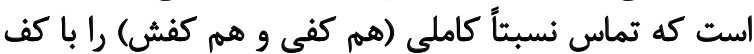

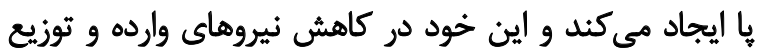
مناسب آن نقش مؤثرى دارد. از طرفى مي دانيم كه ني انتقال امواج
حداكثر نيروى عمودى عكس العمل زمين مشخص شد كئ كه در

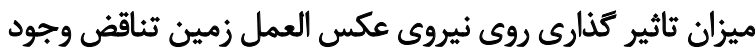

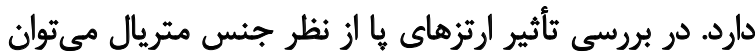

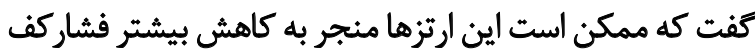

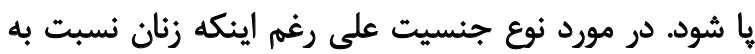

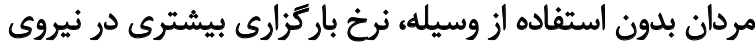

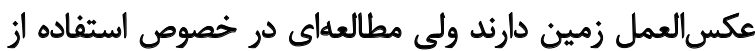
كفى و تأثير جنسيت يافت نشد.

همجنين مشخصات مربوط به هر مقاله نهايىشده كه شامل

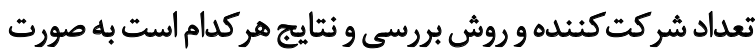

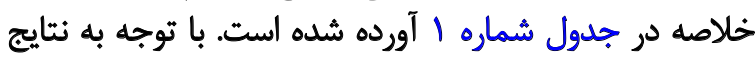

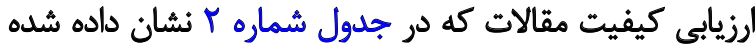

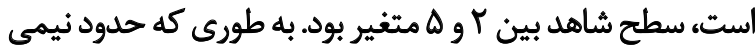

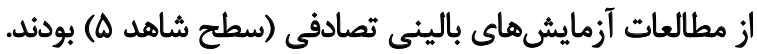

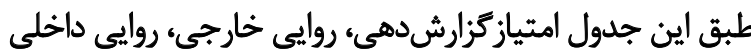

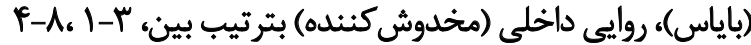

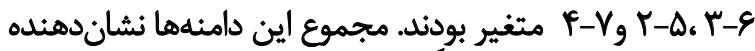

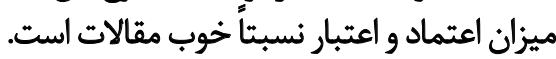

امتياز نهايى ناشى از ارزيابى كيفيت مقالات در سطوح مختلف

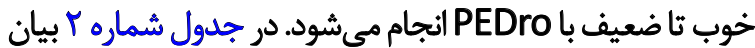

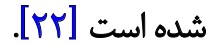
بحث هدف از اين مطالعه، بررسى تغييرات در نيروى عكسالعمل توليد

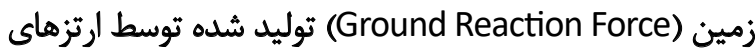

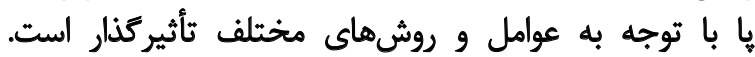
درحقيقت تعيين تأثير مؤلفههاى مختلف بر GRF درو ترائ ما رادر 
جدول r. بررسى كيفيت مقالات

\begin{tabular}{|c|c|c|c|c|c|c|c|}
\hline 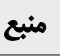 & مؤلف & سطح شاهد & كزّارش دهى & روايي خارجى & روائى داخلى (باياس) & روايي واخلي (مخدوش كثنده) & امتياز PEDRO \\
\hline [rT] & King & $ه$ & $\checkmark$ & $r$ & $\Delta$ & $\Delta$ & 6 (good) \\
\hline [६] & Yung- hui & $ه$ & $\checkmark$ & $r$ & $\Delta$ & $\Delta$ & 5 (fair) \\
\hline$\left[\varphi_{*}\right]$ & Laughton & $r$ & $\checkmark$ & r & $\Delta$ & 8 & 7 (good) \\
\hline [ץג] & Maclean & r & $\checkmark$ & r & $\Delta$ & $\Delta$ & 6 (good) \\
\hline [rV] & Sloss & $\Delta$ & $\Delta$ & $r$ & $r$ & r & 6 (good) \\
\hline$[\mathrm{VV}]$ & Nester & $\Delta$ & 8 & $r$ & $\Delta$ & $\varphi$ & 6 (good) \\
\hline$[\mathrm{HW}]$ & Chiu & $r$ & $\wedge$ & $r$ & r & $ه$ & 4 (fair) \\
\hline$\left[\mathbb{H}^{*}\right]$ & Eslami & $r$ & $\Delta$ & r & $\Delta$ & $\Delta$ & 4 (fair) \\
\hline [r9] & Shiba & r & 8 & $r$ & f & $r$ & 3 (poor) \\
\hline$[\mathrm{IF}]$ & Lin & $\Delta$ & $\Delta$ & $r$ & $r$ & r & 5 (fair) \\
\hline$[r \varepsilon]$ & Creaby & $r$ & $ه$ & $r$ & $r$ & $\Delta$ & 5 (fair) \\
\hline$[r)]$ & MacLean & $r$ & 8 & $r$ & F & $\Delta$ & 5 (fair) \\
\hline [ro] & Sobel & $\Delta$ & $r$ & $r$ & f & $r$ & 4 (fair) \\
\hline$\left[\varphi_{\bullet}\right]$ & Razaghi & r & 8 & 1 & $r$ & p & 5 (fair) \\
\hline$[r \%]$ & Munderman & $\Delta$ & $r$ & $r$ & $\Delta$ & i & 6 (good) \\
\hline$\left[F^{*}\right]$ & Park & $r$ & $\checkmark$ & $r$ & $\Delta$ & 8 & $V$ (good) \\
\hline [ro] & Alirezaee & $r$ & 8 & $r$ & $f$ & $\Delta$ & 6 (good) \\
\hline$[(4)]$ & Tenten & $\Delta$ & $\Delta$ & $r$ & f & $r$ & 3 (poor) \\
\hline$[\pi]$ & Rome & $r$ & 8 & $r$ & $r$ & f & 3 (poor) \\
\hline$[m]$ & Voloshin & $\Delta$ & $\Delta$ & $r$ & $r$ & $\Delta$ & 5 (fair) \\
\hline [rV] & Miller & $r$ & 8 & $r$ & $r$ & i & 4 (fair) \\
\hline
\end{tabular}

هي از يوشيدن كفىهاى قالب گيرىشده كزارش شده است، اما

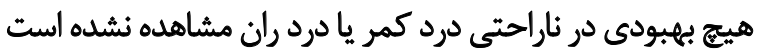

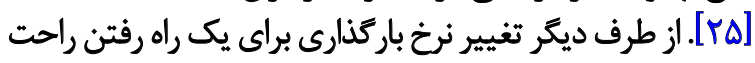

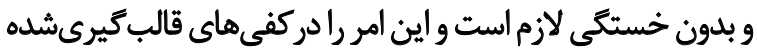

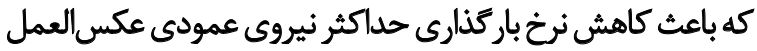

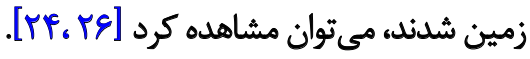

برخلاف اين مطالعات، اسلوس هدر تحقيق خوداعلام كرد يوشيدن

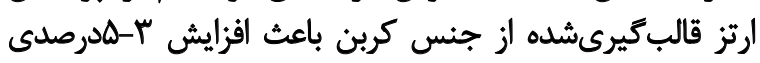

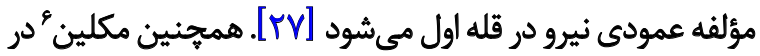
بررسى تأثير قالب نيرى دوى روليرل ميروى عكسالعمل زمين، تغييرات

5. Sloss

6. MacLean

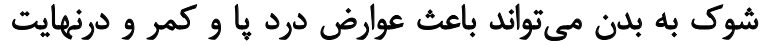

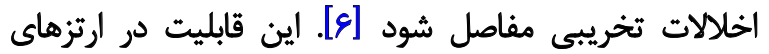

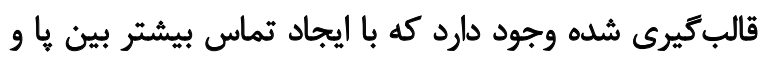
ارتز باعث افزايش جذب شوى شوى و كاهش

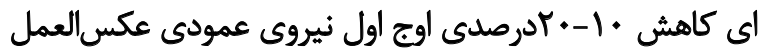

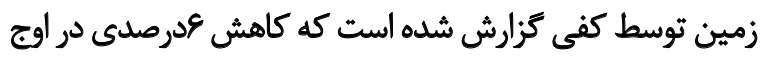

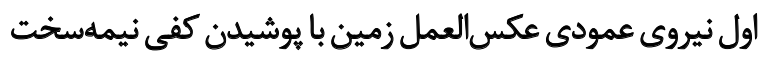

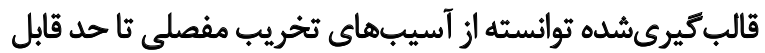

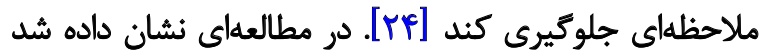

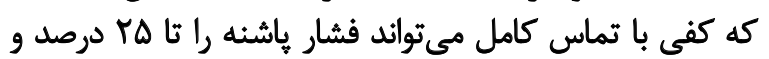

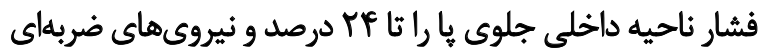

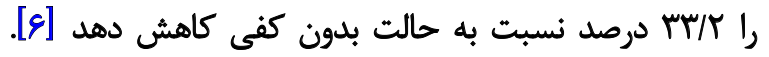

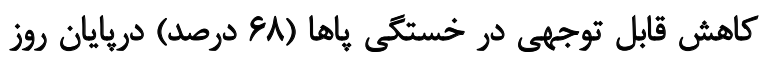


جنس كفى از عواملى است كه در تغيير نيروهاى عكسالعمل

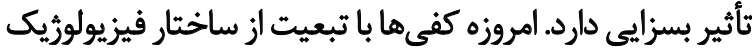

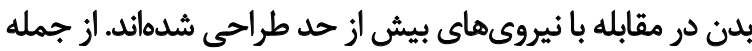

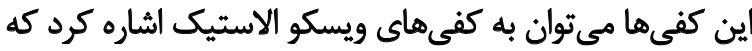

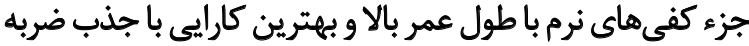

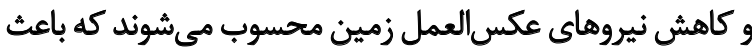

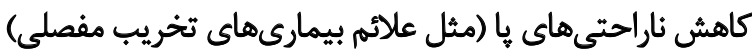

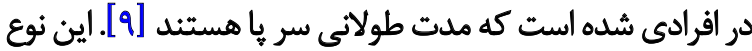

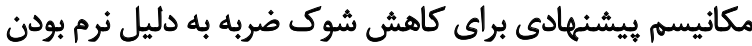

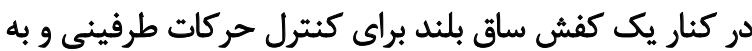

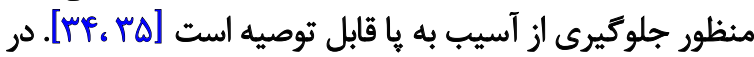

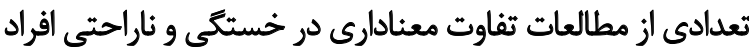

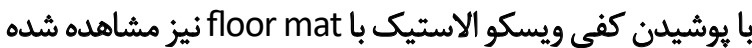

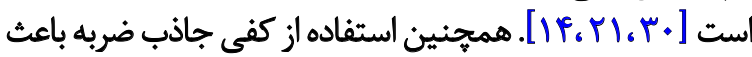

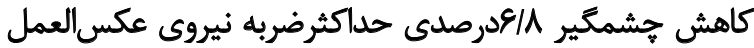

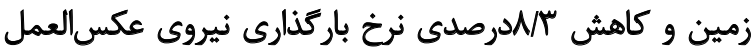

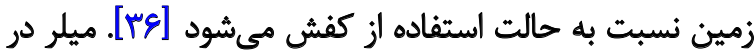

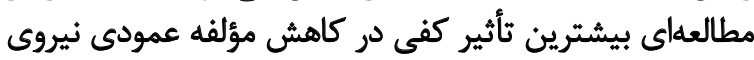

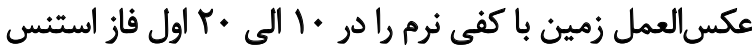

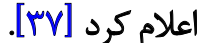

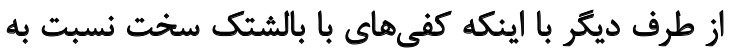

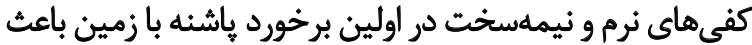

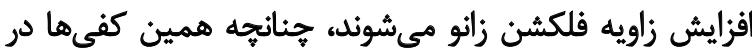

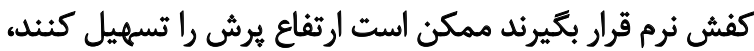

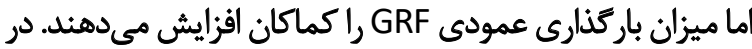

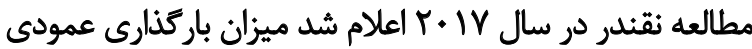

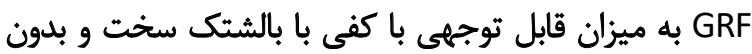

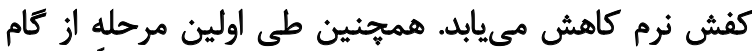

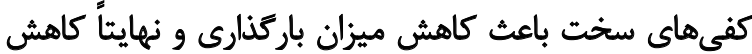

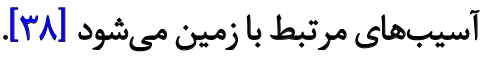

در مقابل اين كزارشات، مطالعاتى اعلام كردند كه مشخصههاى نيروى عكس العمل زمين در مقايسه بين استفادها زاز كفى هائ ويسكو

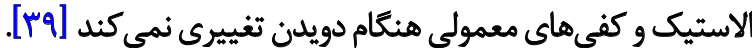

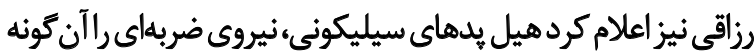

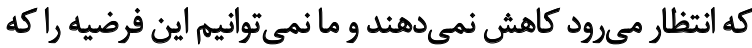

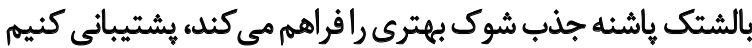

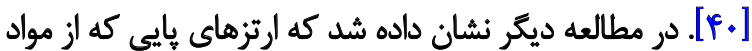

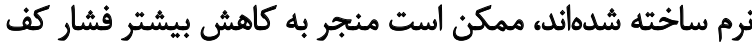

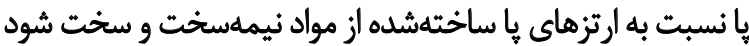

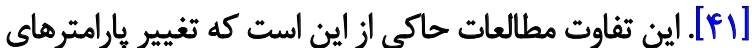

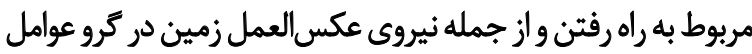

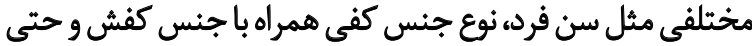
طول عمر جنس كفى هاى نرم است نئ.
معنى دارى در حداكثر نيروى ضربهاى و نرخ باركذارى با استفاده

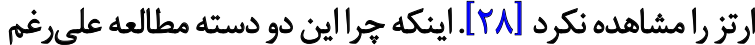

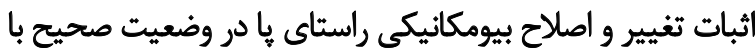

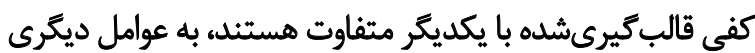

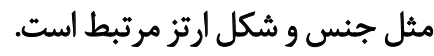
تأثير يست بر نيروى عكس العمل زميين

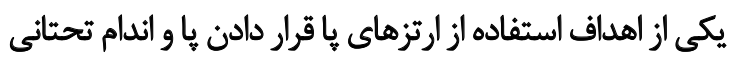

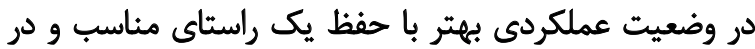

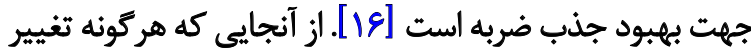

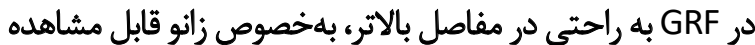

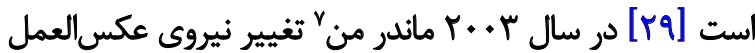

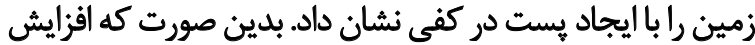

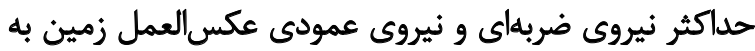

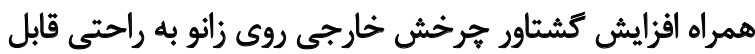

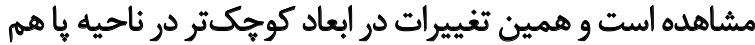

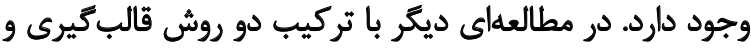

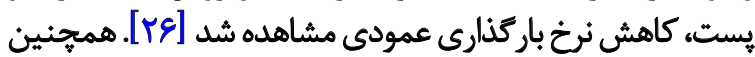

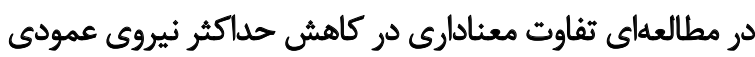

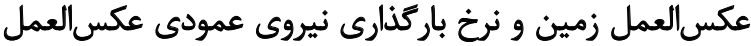

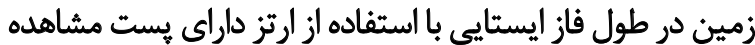

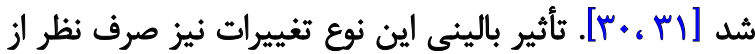

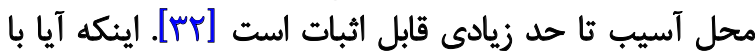

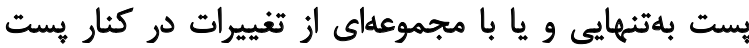

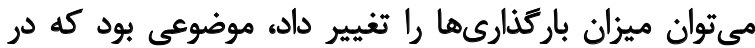

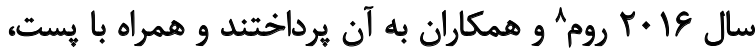

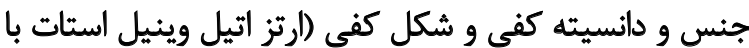

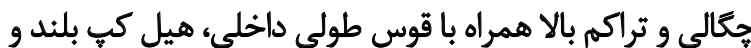

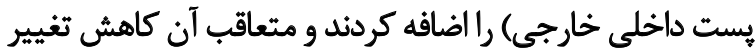

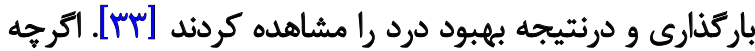

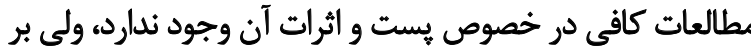

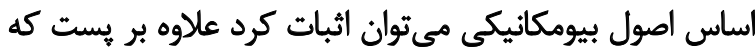

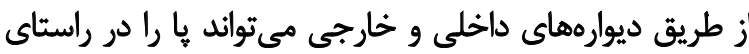

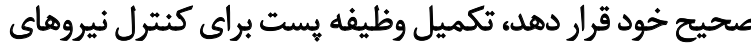

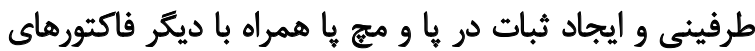

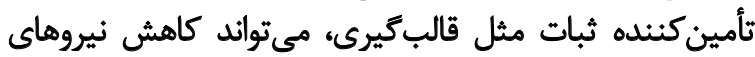

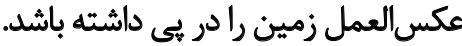
ثأثير مواد نرم بر نيروى عكس العمل زمين

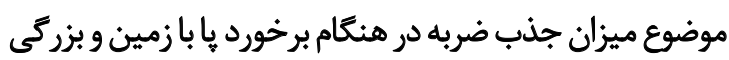

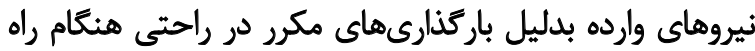

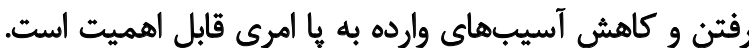

\section{Maunder man}

8. Rome 


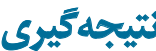

تعيين تأثير مؤلفههاى مختلف بر GRF در كف به با مى تواند ما را

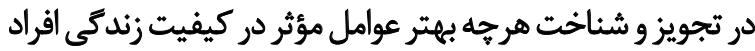

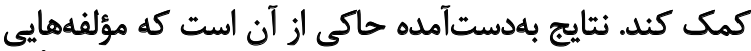

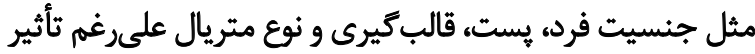

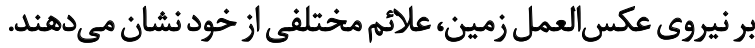

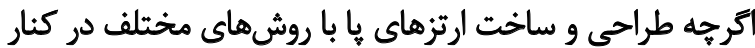

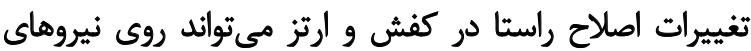

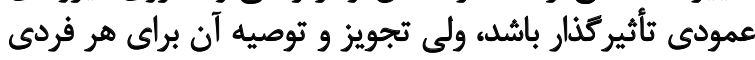

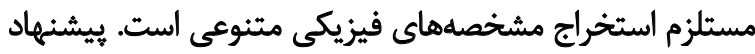

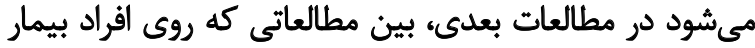

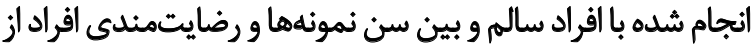

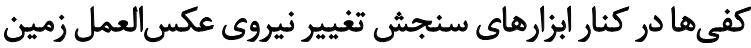

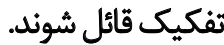

ملاحظات اخلاقي

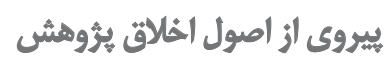

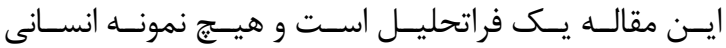

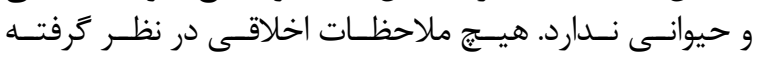

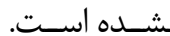

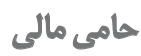

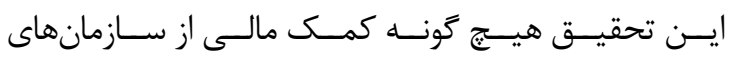

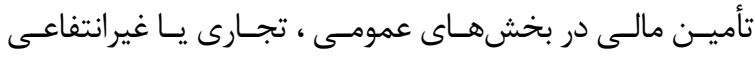

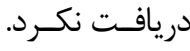

مشاركت نويسند مكان

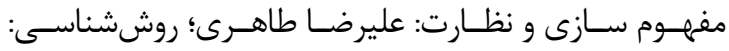

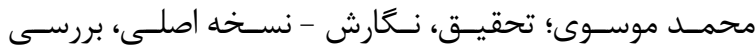

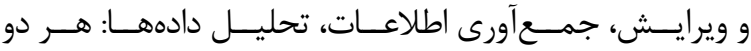

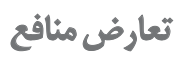

نويسند كان هيج كَونه تضاد منافع ندارند

$$
\text { تشيكر و قبدرواني }
$$

ما از خانم رحمانى ، مسئول كتابخانه دانشكده علوم توانبخشى، براى كمك در جستجوى دقيقتر منابع ، قدردانى مى كنيم.
ثأثير جنسيث فرد بر ثيروى عكس العمل زمين

اترجه يارامترهاى راه رفتن در مرد و زن با هم تا حدى

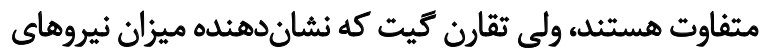

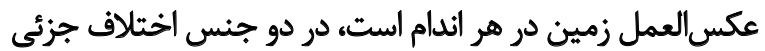

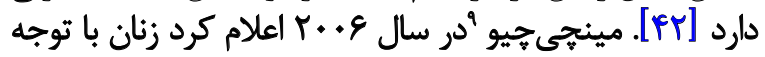

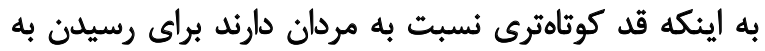

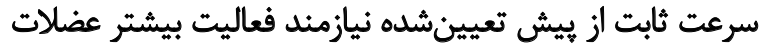

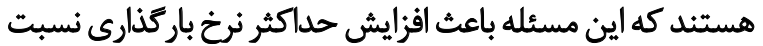

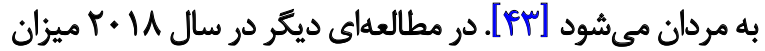

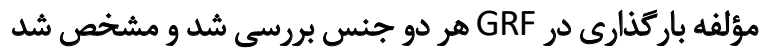

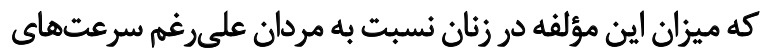

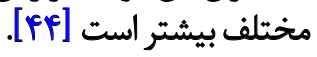

به عبارت ديكر فارغ از استفاده از كفى، زنان نسبت به مردان

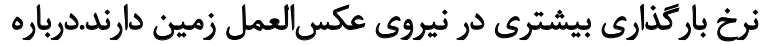

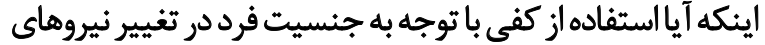

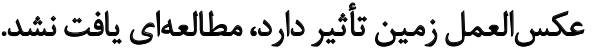

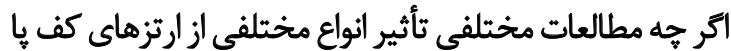

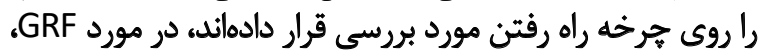

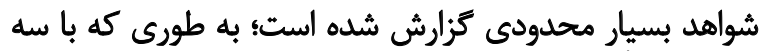

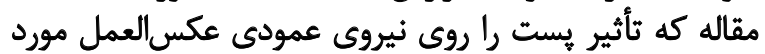
بررسى قرار داده؛ نمى توان به نتيجه قطعى دست ديت يافت.

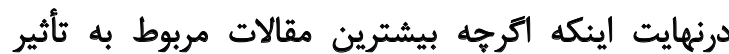

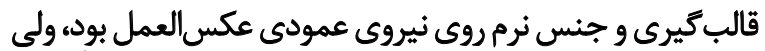

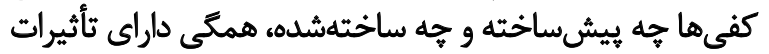

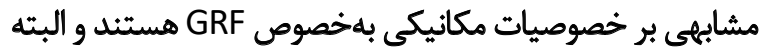

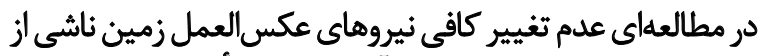

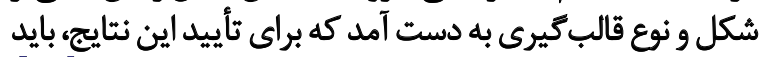

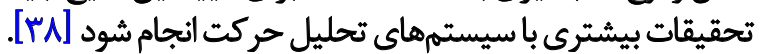

درباره اينكه خرا بسيارى از افراد كه ملزم به استفاده از ارتزهاى

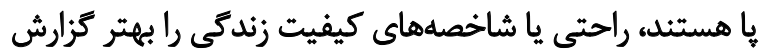

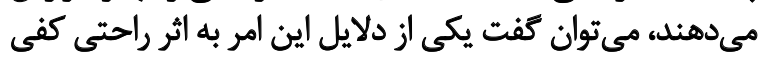

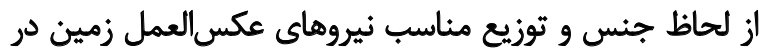

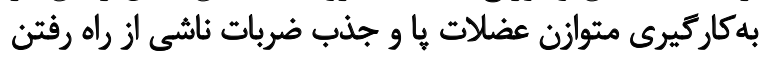

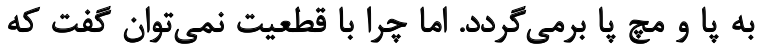

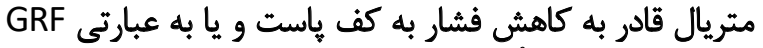

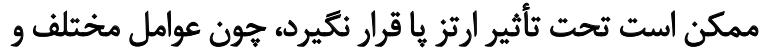

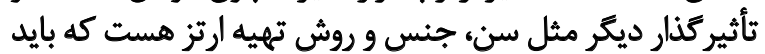

$$
\text { مورد مطالعه بيشتر قرار كيرند. }
$$

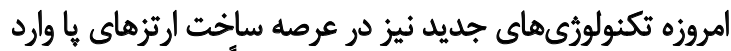

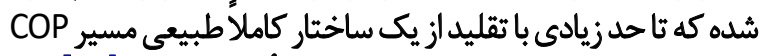

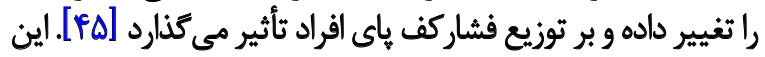

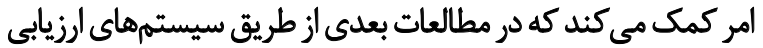
روز دنيا به بررسى همزمان اين عوامل يرداخته شود. بعلي إطريت 


\section{References}

[1] Kharb A, Saini V, Jain YK, Dhiman S. A review of gait cycle and its parameters. IJCEM International Journal of Computational Engineering \& Management. 2011; 13:78-83. https://www.ijcem. org/papers72011/72011_14.pdf

[2] Whittle MW. Generation and attenuation of transient impulsive forces beneath the foot: A review. Gait \& Posture. 1999; 10(3):264-75. [DOI:10.1016/S0966-6362(99)00041-7]

[3] Haim A, Rozen N, Wolf A. The influence of sagittal center of pressure offset on gait kinematics and kinetics. Journal of Biomechanics. 2010; 43(5):969-77. [DOI:10.1016/j.jbiomech.2009.10.045] [PMID]

[4] Cook TM, Farrell KP, Carey IA, Gibbs JM, Wiger GE. Effects of restricted knee flexion and walking speed on the vertical ground reaction force during gait. Journal of Orthopaedic \& Sports Physical Therapy. 1997; 25(4):236-44. [DOI:10.2519/ jospt.1997.25.4.236] [PMID]

[5] Ferber R, Davis IM, Hamill J, Pollard CD, McKeown KA. Kinetic variables in subjects with previous lower extremity stress fractures. Medicine \& Science in Sports \& Exercise. 2002; 34(5):S5. [DOI:10.1097/00005768-200205001-00025

[6] Yung-Hui L, Wei-Hsien H. Effects of shoe inserts and heel height on foot pressure, impact force, and perceived comfort during walking. Applied Ergonomics. 2005; 36(3):355-62. [DOI:10.1016/j.apergo.2004.11.001] [PMID]

[7] Cham MB, Safaeepour Z, Ghasemi MS, Yegane MZ, Forogh B, Sanjari MA. The effect of rocker shoe on the ground reaction force parameters in patients with rheumatoid arthritis. Iranian Rehabilitation Journal. 2015; 13(1):61-7. http://eprints.iums. ac.ir/4993/

[8] Hreljac A, Marshall RN, Hume PA. Evaluation of lower extremity overuse injury potential in runners. Medicine \& Science in Sports \& Exercise. 2000; 32(9):1635-41. [DOI:10.1097/00005768200009000-00018] [PMID]

[9] Aguinaldo A, Mahar A. Impact loading in running shoes with cushioning column systems. Journal of Applied Biomechanics. 2003; 19(4):353-60. [DOI:10.1123/jab.19.4.353]

[10] Daryabor A, Saeedi H, Yazdani M, Ghasemi MS, Nabavi H, Mohammadpour A, et al. The effect of standard and beveled heels of orthopedic shoe on vertical ground reaction forces during walking in healthy subjects. Archives of Rehabilitation. 2013; 14(3):47-55. https://rehabilitationj.uswr.ac.ir/article-1-1285-en.pdf

[11] Bobbert MF, Yeadon MR, Nigg BM. Mechanical analysis of the landing phase in heel-toe running. Journal of Biomechanics. 1992; 25(3):223-34. [DOI:10.1016/0021-9290(92)90022-S]

[12] Mills K, Blanch P, Chapman AR, McPoil TG, Vicenzino B. Foot orthoses and gait: A systematic review and meta-analysis of literature pertaining to potential mechanisms. British Journal of Sports Medicine. 2010; 44(14):1035-46. . [DOI:10.1136/ bjsm.2009.066977] [PMID]

[13] Marras W, Lavender S, Leurgans S, Fathallah F, Ferguson S, Allread $G$, et al. Biomechanical risk factors for occupationally related low back disorders. Journal of Safety Research. 1996; 4(27):271. [DOI:10.1016/S0022-4375(97)81191-7]
[14] Lin YH, Chen CY, Cho MH. Influence of shoe/floor conditions on lower leg circumference and subjective discomfort during prolonged standing. Applied Ergonomics. 2012; 43(5):965-70. [DOI:10.1016/j.apergo.2012.01.006] [PMID]

[15] Heiderscheit B, Hamill J, Tiberio D. A biomechanical perspective: do foot orthoses work? British Journal of Sports Medicine. 2001; 35(1):4-5. [DOI:10.1136/bjsm.35.1.4] [PMID] [PMCID]

[16] Bird AR, Bendrups AP, Payne CB. The effect of foot wedging on electromyographic activity in the erector spinae and gluteus medius muscles during walking. Gait \& Posture. 2003; 18(2):81-91. [DOI:10.1016/S0966-6362(02)00199-6]

[17] Nester C, Van Der Linden M, Bowker P. Effect of foot orthoses on the kinematics and kinetics of normal walking gait. Gait \& Posture. 2003; 17(2):180-7. [DOI:10.1016/S09666362(02)00065-6]

[18] Akbari M, Saeedi H, Babaee T. The effect of lateral wedge and medial arch support on displacement of ground reaction force in patients with knee osteoarthritis. Archives of Rehabilitation. 2016;17(1):74-83. [DOI:10.20286/jrehab-170172]

[19] Shamsi F, Tabatabai Ghomshe F, Bahramizade M, Mousav ME, Rezasoltani P. [Effects of lateral heel wedges and lateral forefoot wedge on the knee adduction moment in healthy male students (Persian)]. Journal of Rehabilitation. 2012; 12(4):27-34. https://rehabilitationj.uswr.ac.ir/browse.php?a_code=A-10$371-1 \& \mathrm{ftxt}=1$

[20] Kim J, Stuart-Buttle C, Marras W. The effects of mats on back and leg fatigue. Applied Ergonomics. 1994; 25(1):29-34. [DOI:10.1016/0003-6870(94)90028-0]

[21] Higgins JP, Thomas J, Chandler J, Cumpston M, Li T, Page MJ, et al., Editor (s). Cochrane handbook for systematic reviews of interventions. Hoboken, New Jersey: John Wiley \& Sons; 2019. [DOI:10.1002/9781119536604]

[22] Eng J, Teasell R, Miller W, Wolfe D, Townson A, Aubut JA, et al. Spinal cord injury rehabilitation evidence: Method of the SCIRE systematic review. Topics in Spinal Cord Injury Rehabilitation. 2007; 13(1):1-10. [DOI:10.1310/sci1301-1] [PMID] [PMCID]

[23] King PM. A comparison of the effects of floor mats and shoe in-soles on standing fatigue. Applied Ergonomics. 2002; 33(5):477-84. [DOI:10.1016/S0003-6870(02)00027-3]

[24] Eslami M, Begon M, Hinse S, Sadeghi H, Popov P, Allard P. Effect of foot orthoses on magnitude and timing of rearfoot and tibial motions, ground reaction force and knee moment during running. Journal of Science and Medicine in Sport. 2009; 12(6):679-84. [DOI:10.1016/j.jsams.2008.05.001] [PMID]

[25] Sobel E, Levitz SJ, Caselli MA, Christos PJ, Rosenblum J. The effect of customized insoles on the reduction of postwork discomfort. Journal of the American Podiatric Medical Association. 2001; 91(10):515-20. [DOI:10.7547/87507315-91-10-515] [PMID]

[26] Mündermann A, Nigg BM, Humble RN, Stefanyshyn DJ Foot orthotics affect lower extremity kinematics and kinet ics during running. Clinical Biomechanics. 2003; 18(3):254-62. [DOI:10.1016/S0268-0033(02)00186-9] 
[27] Sloss R. The effects of foot orthoses on the ground reaction forces during walking. Part 1. The Foot. 2001; 11(4):205-14. [DOI:10.1054/foot.2001.0713]

[28] MacLean C, Davis IM, Hamill J. Influence of a custom foot orthotic intervention on lower extremity dynamics in healthy runners. Clinical Biomechanics. 2006; 21(6):623-30. [DOI:10.1016/j. clinbiomech.2006.01.005] [PMID]

[29] Norasteh AA, Emami S, Shamsi Majelan A. [Kinetic and kinematic variables in middle-aged women with normal and genu varum knee angle with emphasis on walking and running activities (Persian)]. Physical Treatments-Specific Physical Therapy Journal. 2014; 4(2):77-82. http://ptj.uswr.ac.ir/article-1-144-en.html

[30] Laughton CA, Davis IM, Hamill J. Effect of strike pattern and orthotic intervention on tibial shock during running. Journal of Applied Biomechanics. 2003; 19(2):153-68. [DOI:10.1123/ jab.19.2.153]

[31] MacLean CL, Davis IS, Hamill J. Short-and long-term influences of a custom foot orthotic intervention on lower extremity dynamics. Clinical Journal of Sport Medicine. 2008; 18(4):338-43. [DOI:10.1097/MJT.0b013e31815fa75a]

[32] Cho NS, Hwang JH, Chang HJ, Koh EM, Park HS. Randomized controlled trial for clinical effects of varying types of insoles combined with specialized shoes in patients with rheumatoid arthritis of the foot. Clinical Rehabilitation. 2009; 23(6):512-21. [DOI:10.1177/0269215508101737] [PMID]

[33] Rome K, Clark H, Gray J, McMeekin P, Plant M, Dixon J. Clinical effectiveness and cost-effectiveness of foot orthoses for people with established rheumatoid arthritis: An exploratory clinical trial. Scandinavian Journal of Rheumatology. 2017; 46(3):187-93. [DOI:10.1080/03009742.2016.1196500] [PMID]

[34] Voloshin A, Wosk J. An in vivo study of low back pain and shock absorption in the human locomotor system. Journal of Biomechanics. 1982; 15(1):21-7. [DOI:10.1016/0021-9290(82)90031-8]

[35] O'Leary K, Vorpahl KA, Heiderscheit B. Effect of cushioned insoles on impact forces during running. Journal of the American Podiatric Medical Association. 2008; 98(1):36-41. [DOI:10.7547/0980036] [PMID]

[36] Creaby MW, May K, Bennell KL. Insole effects on impact loading during walking. Ergonomics. 2011; 54(7):665-71. [DOI:10.10 80/00140139.2011.592600] [PMID]

[37] Miller CD, Laskowski ER, Suman VJ, editors. Effect of corrective rearfoot orthotic devices on ground reaction forces during ambulation. Mayo Clinic Proceedings; 1996; 71(8):757-62. [DOI:10.1016/S0025-6196(11)64840-4] [PMID]

[38] Alirezaei Noghondar F, Bressel E. Effect of shoe insole density on impact characteristics and performance during a jump-landing task. Footwear Science. 2017; 9(2):95-101. [DOI:10.1080/19424 280.2017.1305003]

[39] Shiba N, Kitaoka HB, Cahalan TD, Chao E. Shock-absorbing effect of shoe insert materials commonly used in management of lower extremity disorders. Clinical Orthopaedics and Related Research. 1995; (310):130-6. [DOI:10.1097/00003086199501000-00021]
[40] Razeghi M, Batt ME. The effect of foot orthoses on selected ground reaction force parameters during ground walking [Internet]. 2013 [Updated 2013]. Available from: https://citeseerx.ist. psu.edu/viewdoc/download?doi=10.1.1.577.4386\&rep=rep1\&t ype $=$ pdf

[41] Tenten-Diepenmaat M, Dekker J, Heymans MW, Roorda LD, Vlieland TPV, Van Der Leeden M. Systematic review on the comparative effectiveness of foot orthoses in patients with rheumatoid arthritis. Journal of Foot and Ankle Research. 2019; 12(1):32. [DOI:10.1186/s13047-019-0338-x] [PMID] [PMCID]

[42] Nasirzade A, Sadeghi H, Mokhtarinia HR, Rahimi A. A review of selected factors affecting gait symmetry. Physical Treatments-Specific Physical Therapy Journal. 2017; 7(1):3-12. [DOI:10.29252/ nrip.ptj.7.1.3]

[43] Chiu MC, Wang MJ. The effect of gait speed and gender on perceived exertion, muscle activity, joint motion of lower extremity, ground reaction force and heart rate during normal walking. Gait \& Posture. 2007; 25(3):385-92. [DOI:10.1016/j.gaitpost.2006.05.008] [PMID]

[44] Park SK, Koo S, Yoon SH, Park S, Kim Y, Ryu JS. [Gender dfferences in ground reaction force components (Korean)]. Korean Journal of Sport Biomechanics. 2018; 28(2):101-8. https://www. koreascience.or.kr/article/JAKO201821464987479.page

[45] Joo JY, Kim YK. [Effects of customized 3D-printed Insoles on the Kinematics of flat-footed walking and running (Korean)]. Korean Journal of Sport Biomechanics. 2018; 28(4):237-44. http:/ /www.papersearch.net/thesis/article.asp?key $=3655349$ 